\title{
L'evolution du rôle des organisations non gouvernementales dans le droit de l'environnement*
}

\author{
Juan Andrés Fuentes Véliz
}

A María

\section{Introduction}

Comme sujet de ce travail, nous avons choisi l'évolution du rôle des organisations non gouvernementales (ci-après «ONG») dans le droit international de l'environnement. Notre hypothèse de travail a été que les ONG ont connu un développement extraordinaire dans le droit de l'environnement ce qui a contribué également à ce que ce domaine évolue tellement dans un temps si court. Mais cela n'était qu'une hypothèse, une affirmation faite sur base de la simple et peu profonde appréciation de ce sujet-ci.

A présent, voici nos constatations. Les origines du droit de l'environnement se situent après la deuxième guerre mondiale car l'histoire nous apprend que c'est malheureusement après des tragédies que nous réagissons. Dans la période de reconstruction et suite à la raréfaction de l'eau, de la faune et de la flore, les pays se sont rendu compte de leur importance. ${ }^{1}$ La Convention de Londres du 12 mai 1954 pour la Prévention de la Pollution des Eaux de la Mer par les Hydrocarbures fut la première ébauche au niveau international d'une législation sur la protection de l'environnement. ${ }^{2}$ Dans le domaine militaire, le Traité de Moscou du 5 août 1963 a essayé d'interdire les

\footnotetext{
* Quisieramos agradecer al profesor Gautier por sus consejos a lo largo de este estudio.

KIss, A. Droit international de l'environnement. Paris: Pedone 2000. Avant cela, au XIXe siècle, les États avaient signé des accords relatifs à la pêche, mais il s'agissait plutôt de délimiter les zones de pêche que de protéger la nature. Il a fallu attendre jusqu'à la deuxième moitié du XXe siècle pour voir une évolution en la matière.

2 Cette convention a été remplacée par la Convention pour la Prévention de la Pollution par les Navires de 1973, modifiée en 1978.
} 
essaies d'armes nucléaires dans l'atmosphère, dans l'espace extra atmosphérique et sous l'eau.

Néanmoins, 1968 est une année où des actions très importantes ont été menées. Le Comité des Ministres du Conseil de l'Europe a adopté le 8 mars la Résolution 68 (4) en vue de protéger l'environnement. Tout de suite, le 6 mai 1968, fut adoptée la Charte Européenne de l'Eau. L'exemple a été suivi en Afrique lorsque les chefs d'État africains ont signé le 15 septembre de la même année une Convention sur la conservation de la nature et des ressources naturelles. L'Organisation des Nations Unies (ci-après "ONU») s'est également engagée dans la tâche écologique. Le 3 décembre 1968, l'Assemblée Générale a prévu la convocation d'une conférence mondiale sur l'environnement pour 1972 à Stockholm. ${ }^{3}$

D'ailleurs, il convient de préciser que grâce à la reconnaissance du droit à un environnement sain comme un droit de l'homme, le droit de l'environnement aura un développement plus rapide. Effectivement, la Charte Africaine des Droits de l'Homme et des Peuples de $1981^{4}$ (ci-après "Charte Africaine») fut la première convention internationale à garantir de façon effective ${ }^{5}$ le droit à un environnement sain ${ }^{6}$ de telle sorte que celui-ci est considéré aujourd'hui comme un droit de l'homme. ${ }^{7}$ La raison est que «l'environnement n'est pas une abstraction, mais bien l'espace où vivent les êtres humains et dont dépendent la qualité de leur vie et leur santé, y compris pour les générations à venir $(\ldots){ }^{8}{ }^{8}$

Dans ce cadre là, les ONG jouent un rôle primordial puisqu'elles permettent une participation plus active des citoyens dans les sujets de société. En effet, l'apparition des

\footnotetext{
3 KIss, supra note 1, p. 30.

4 Voy. Wikypédia L'Encyclopédie Libre, disponible sur <http://fr.wikipedia.org/wiki/Charte_africaine_ des_droits_de_l'homme_et_des_peuples>. Cette convention fut adoptée par la dix-huitième Conférence des Chefs d'État et de Gouvernement, juin 1981, Nairobi, Kenya. 49 des 52 membres de l'Organisation de l'Unité Africaine l'ont ratifié. BRETON-Legoff, G. «Policy paper: bourse canadian consortium on human security 2003-2004», disponible sur <http://www.humansecurity.info/sites/cchs/files/pdfs/Fellow\%20Papers/ Policy_brief/LeGoff_Policy_Paper.pdf>. Selon les recherches de Breton-Le Goff, les ONG ont favorisé la naissance de cette charte, «Le rôle des ONG a été 1) de sensibiliser les juristes africains à la problématique des droits de l'homme en Afrique, 2) de les aider à rédiger un projet de texte qui soit adapté à la réalité africaine et intègre les différents aspects culturels, 3) de favoriser la ratification des traités...».

Voy. Commission Africaine des Droits de l'Homme et des Peuples, Lignes directrices, disponible sur $<$ http://www.achpr.org/francais/_info/guidelines_communications_fr.html>. Nous parlons d' "effectivité» ici parce que la Charte Africaine permet aux individus comme tels aller devant une voie quasi juridictionnelle internationale (la Commission Africaine des Droits de l'Homme et des Peuples).

6 L'initiative africaine sera suivie par d'autres blocs régionaux comme les pays américains via la signature du Protocole Additionnel de la Convention Américaine des Droits de l'Homme (17 novembre 1988), Art. 11.

Le droit à un environnement sain est un droit reconnu par la doctrine comme un des «droits de solidarité» (avec le droit au développement, entre autres). La reconnaissance de ce droit comme droit de l'homme a été promue surtout par les pays du Tiers-Monde.

8 Voy. Licéité de la Menace ou de l'Emploi d'Armes Nucléaires, CIJ Recueil 1996, pp. 241-242, \$ 29.
} 
ONG a conduit à traiter des problèmes de dimension collective, ayant un sens pour la société dans son ensemble. Grâce au travail des ONG, les citoyens peuvent s'informer sur les problématiques actuelles dans différents domaines et — s'ils le souhaitent-influencer l'ordre des priorités de l'agenda établi par leurs autorités. ${ }^{9}$ De cette façon, ils peuvent intervenir — de manière indirecte—dans le decision making process.

Ensuite, le rôle politique croissant des ONG sur la scène internationale est un fait incontestable. Leurs activités notamment en tant qu'amicus curiae de cours supranationales, ${ }^{10}$ intervenants dans la rédaction des conventions ${ }^{11}[\ldots]$ ne font qu'enrichir de plus en plus le droit international. Leur importance est aussi reflétée statistiquement. Selon le Yearbook of International Organisations, il y a avait 3733 ONG en 1972. En 2005 , ce chiffre est monté à $58859 .{ }^{12}$

Enfin, et plus particulièrement en droit de l'environnement, les ONG sont directement impliquées dans le développement de la protection de l'environnement ${ }^{13} \mathrm{car}$ c'est grâce à leur travail effectué ces dernières années qu'elles ont réussi à attirer l'attention des autorités étatiques du monde entier. Ainsi, les États, ${ }^{14}$ sujets de droit par excellence, consacrent le droit à l'environnement dans leurs textes constitutionnels. ${ }^{15}$ Ils concluent également des traités entre eux mais aussi avec d'autres sujets de droit international; ${ }^{16}$ créent des institutions régionales, et programmes universels de

\footnotetext{
$9 \quad$ En fait, le travail éducatif accompli par les ONG en faveur de la population quant à la conscientisation des risques que comportent chacune de leurs actions contre l'environnement est très important.

10 G. Cornu. Vocabulaire juridique. Paris: PUF 2004, cité dans I. SoumY, «L'accès des organisations non gouvernementales aux juridictions internationales», disponible sur $<$ http://www.unilim.fr/theses/2005/droit/ 2005limo0502/soumy_i.pdf>. Amicus curiae se traduit littéralement par «ami de la Cour» et "désigne la qualité de consultant extraordinaire et d'informateur bénévole en laquelle une juridiction saisie invite à l'audience afin de fournir, en présence de tous les intéressés «toutes les informations propres à éclairer».

11 Breton-Legoff, supra note 4. Les ONG «ont contribué à la rédaction de la Convention du Conseil de l'Europe contre la Torture et de son mécanisme de visite in situ».

12 Voy. Union of International Associations. Yearbook of International Organizations, disponible sur <http:// www.uia.org/statistics/organizations/types-2004.pdf>.

13 Voy. A. Kiss et D. Shelton. Traité de droit européen de l'environnement. Paris: Frison-Roche, 1995. Les auteurs citent la définition de l'environnement contenue dans l'article 2 de la directive du Conseil de la Communauté Economique Européenne du 27 juin 1967. «On entend par environnement l'eau, l'air et la terre d'une part, ainsi que les rapports de ces éléments entre eux, et avec tout organisme vivant».

14 Dans le présent travail, nous utiliserons l'État comme synonyme de pays, sachant qu'il ne s'agit pas de deux concepts tout à fait identiques. «Pays» désigne un territoire d'étendue restreinte tandis qu' "État» est une entité gouvernant l'organisation d'un pays qui est délimité par des frontières territoriales et constitué d'institutions lui assurant un pouvoir suprême.

15 Kiss et as., supra note 13, p. 47. Pratiquement toutes les constitutions adoptées depuis 1970 proclament ce droit.

16 Au niveau international, voy. par exemple la Convention-Cadre des Nations Unies sur les Changements Climatiques du 15 mai 1992. Au niveau régional, la Convention de Berne relative à la Conservation de la Vie Sauvage et du Milieu Naturel de l'Europe du 19 septembre 1979.
} 
défense de l'écologie; ${ }^{17}$ réalisent à travers des organisations internationales, des conférences pour discuter de nouveaux défis; ${ }^{18}$ octroient actuellement de plus en plus de prérogatives aux ONG en Droit Internationa $\mathrm{l}^{19} \mathrm{en}$ reconnaissant que tous les peuples ont droit à un environnement global satisfaisant, propice à leur épanouissement ${ }^{20}$ et que les États eux-mêmes ont l'obligation de protéger l'environnement. Pour ces raisons, il nous a paru important d'étudier l'évolution du rôle des ONG dans le droit de l'environnement.

La première partie de notre travail abordera brièvement les origines des ONG. Nous tenterons aussi de définir ce type d'organisations afin de les différencier des autres acteurs non-étatiques (sociétés transnationales et organisations internationales). Pour donner au présent travail une optique moins théorique, nous ferons des références spécifiques à Greenpeace. ${ }^{21}$ Ensuite, sur la base de la théorie la plus accueillie actuellement par la doctrine et sur base de l'accord passé entre la Suisse et la Fédération Internationale des Sociétés de la Croix-Rouge et du Croissant-Rouge, nous étudierons si les ONG peuvent être qualifiées de sujets de Droit International.

La deuxième partie constatera l'évolution des divers rôles assumés par les ONG dans le droit de l'environnement. Au départ simples groupes de pression, les ONG ont

17 Kiss et as., supra note 13, pp. 121-122. Par exemple, l'Agence Européenne de l'Environnement, organe de l'Union Européenne, a été crée par décision du Conseil de l'Europe du 7 mai 1990. D'autres pays non membres de l'Union peuvent être parties de cet organe. Au niveau mondial et après une recommandation de la Conférence de Stockholm, le programme le plus important dans ce domaine est le Programme des Nations Unies pour l'environnement (PNUE), créé en 1972. "Le rôle premier du P.N.U.E. est d'inciter les autres institutions à agir». Le Président Jacques Chirac a lancé un appel pour renforcer la gouvernance internationale dans ce domaine. «Nous appelons à transformer le Programme des Nations Unies pour l'Environnement en une véritable Organisation internationale à composante universelle à l'image de l'Organisation mondiale de la Santé» a-t-il ajouté. Etudier la pertinence de cette proposition est recommandé. "Appel pour une Véritable ONU de l'Environnement», Metro, 5 février 2007, p. 5.

18 Nous pouvons citer à cet égard la Conférence de Stockholm sur l'Environnement Humain de 1972, promue par l'ONU.

19 Les ONG peuvent saisir la Commission Africaine des Droits de l'Homme et des Peuples, la Commission Interaméricaine de Droits de l'Homme, la Cour Européenne des Droits de l'Homme, etc. Les ONG forment partie, à la demande des gouvernements, des délégations nationaux qui négocient et adoptent traités. «For example, the U.S. delegation to the conference that negotiated the 2000 Convention on the Conservation and Management of Highly Migratory Fish Stocks in the Western and Central Pacific Ocean included representatives not only from the U.S. Department of State and other federal agencies, but representatives from environmental NGOs and the U.S. fishing industry as well». HolLIs, D. «Private actors in public international law: amicus curiae and the case for the retention of state sovereignty", disponible sur <http://www. bc.edu/bc_org/avp/law/lwsch/journals/bciclr/25_2/04_FMS.htm>.

20 Voy. Charte Africaine des Droits de l'Homme et des Peuples, disponible sur <http://www.achpr.org/francais/_info/charter_fr.html>, Art. 24.

21 Voy. Greenpeace. What we do, disponible sur <http://www.greenpeace.org/international/campaigns>. Greenpeace est une organisation qui est connue par ses campagnes pour arrêter les tests nucléaires ainsi que mettre fin à la chasse de baleines. Actuellement, l'organisation s'occupe aussi d'autres problèmes environnementaux tels que le réchauffement climatique, la destruction des forets, l'ingénierie génétique et le pouvoir nucléaire. 
évolué vers des rôles plus dynamiques comme leur participation en qualité de requérants dans des litiges devant des cours internationales ou d'observateurs vis-à-vis des instruments internationaux les plus importants dans cette matière.

\section{Aspects generaux}

\subsection{Origines des organisations non gouvernementales}

Les origines des organisations non gouvernementales remontent au XIXe siècle ${ }^{22}$ quand Henry Dunant, effrayé par les horreurs de la bataille de Solferino a pris l'initiative de mobiliser les femmes du village pour secourir les blessés et les mourants abandonnés par leurs propres troupes sur le champ de bataille. Suite à cela, il a publié "Un souvenir de Solférino» (1862), qui eut un grand impact en Europe. Dans son livre, Henry Dunant écrivit notamment:

N'y aurait-il pas moyen, pendant une époque de paix et de tranquillité, de constituer des sociétés de secours dont le but serait de faire donner des soins aux blessés, en temps de guerre, par des volontaires zélés, dévoués et bien qualifiés pour une pareille œuvre? ${ }^{23}$

C'est à partir de ces idées que le mouvement d'aide humanitaire de la Croix-Rouge est né, essaimant de pays en pays à mesure que des membres des communautés locales se rassemblaient pour créer une société, élire un conseil de direction bénévole et recruter des volontaires. ${ }^{24}$ Puis, cet exemple sera suivi par d'autres personnes autour du monde et dans autres domaines comme l'écologie ou les droits de l'homme.

Ainsi, par exemple, les origines de Greenpeace sont liées à la formation du Comité Don't Make a Wave 25 à Vancouver-British Columbia par un groupe de pacifistes et écologistes des États-Unis et du Canada en $1970 .{ }^{26}$ Le groupe d'activistes s'était embarqué à bord du Phyllis Cormack pour rejoindre le site des essais nucléaires d'Amchitka en Alaska. ${ }^{27}$ Leur objectif était d'empêcher l'explosion de la bombe par les

22 De l'avis de Monsieur Carreau ce phénomène associatif est très ancien, en remontant au XVIII siècle. C'est-à-dire que les ONG précèdent les organisations internationales intergouvernementales. Voy. CARREAU, D. Droit international. Paris: Pedone, 2001.

23 Voy. Fédération Internationale des Sociétés de la Croix -Rouge et du Croissant-Rouge. «L'origine du volontariat Croix-Rouge», disponible sur <http://www.ifrc.org/fr/voluntee/origin.asp>.

24 Voy. Memo, le Site de l'Histoire, Comité International de la Croix-Rouge (CICR), disponible sur <http:// www.memo.fr/article.asp?ID=REG_GEN_INT_005>.

25 Greenpeace. About Greenpeace. The history of Greenpeace, disponible sur <http://www.greenpeace.org/international/about/history>. Le nom du Comité a été tiré d'un slogan utilisé durant les protestations contre les tests nucléaires menés par les États-Unis en 1969.

26 Greenpeace. About Greenpeace. The history of Greenpeace. The founders, disponible sur <http://www. greenpeace.org/international/about/history/founders $>$.

27 Greenpence, supra note 21. Amchitka c'est une île localisée in Alaska et elle était le refuge pour 3000 loutres marines, des faucons, des aigles et d'autres espèces des animaux en danger d'extinction. 
États-Unis. ${ }^{28}$ L'engagement de ces militants d'un genre nouveau a été couronné de succès. Dès 1972, les États-Unis ont cessé les essais nucléaires atmosphériques. ${ }^{29}$ Greenpeace était née. ${ }^{30}$

\subsection{Définition}

Il est toujours difficile de donner une définition à une institution juridique, spécialement les ONG à cause de la disparité des entités qui entrent sous ce vocable mais nous tenterons néanmoins d'en donner une, compte tenu des caractéristiques principales des organisations non gouvernementales.

Tout d'abord, l'expression d' «organisation non gouvernementale» est apparue en 1945 dans le vocabulaire international, dans la Charte des Nations Unies. ${ }^{31}$ Par la suite, ce terme a été progressivement précisé par la doctrine et la pratique des relations internationales. ${ }^{32}$

2.2.1. Les ONG sont des entités de droit privé: Elles ne sont créées par voie législative. Ce sont les particuliers, qui par leur propre volonté, décident de fonder une ONG en conformité avec les lois d'un certain pays. Les ONG établissent leur siège social dans cet État et peuvent avoir des filiales dans d'autres pays. ${ }^{33}$

En réalité, l'organisation Greenpeace se compose de Greenpeace International (Stichting Greenpeace Council) ${ }^{34}$ aux Pays-Bas et d'autres filiales situés partout dans le monde.

28 Id.

29 Greenpence. About Greenpeace. Greenpeace victories. Disponible sur <http://www.greenpeace.org/international/about/victories>. Amchitka fut déclarée après un sanctuaire des oiseaux.

30 Greenpeace, supra note 24. Tout de suite, le groupe a changé le nom de son mouvement par un nom qui exprime mieux sa double préoccupation: l'environnement (green) et le pacifisme (peace).

31 Voy. Charte des Nations Unis, Art. 71, disponible sur <http://www.un.org/french/aboutun/charte>. Il faut dire que Greenpeace International jouit d'un statut consultatif devant le Conseil Economique et Social des Nations Unies.

32 Voy. Webhumanitaire. Toutsavoirsurlesorganisationsnongouvernementales. Disponiblesur $<\mathrm{http} / /$ membres. lycos.fr/webhumanitaire/ONG.htm>.

33 Voy. Mariño Menéndez, F. Derecho internacional público. Madrid: Trotta 1999. Dans chaque pays, le gouvernement peut demander aussi l'enregistrement administratif des ONG et établir certaines conditions pour qu'elles puissent opérer. En Hongrie des conditions raisonnables sont requis «[...], une ONG doit avoir un conseil d'administration, au moins 10 membres et faire une déclaration d'intention». Kiss et as., supra note 13, pp. 535-536. Mais aussi, il y a des pays où ils existent des lois qui restringent ouvertement l'action des ONG. Nous pouvons citer le cas du Pérou, la Russie, la Biélorussie et le Zimbabwe. Martin, C. et as., "Hugo Chávez también quiere aprobar ley para controlar donaciones de ONGs», La República, 2 Novembre 2006. Disponible sur <http://www.larepublica.com.pe/index.php?option=com_content\& task=view\&id=129651\&Itemid=0>.

34 Greenpeace. About Greenpeace. How is Greenpeace structured. Disponible sur <http://www.greenpeace. org/international/about/how-is-greenpeace-structured>.

Greenpeace. About Greenpeace. Our mission. Disponible sur <http://www.greenpeace.org/international/ about/our-mission>. Le rôle de Greenpeace International est de faciliter le processus de planification et de 
Greenpeace est aujourd'hui présente dans 41 pays. Ces filiales nationales et régionales ont des licences pour utiliser le nom «Greenpeace». ${ }^{35}$

2.2.2. Les ONG sont des entités autonomes des États: Conséquence intimement lié à leur première caractéristique, il importe de souligner l'autonomie des organisations non gouvernementales. Celles-ci «are essentially voluntary self-governing bodies and are not therefore subject to direction by public authorities». ${ }^{36}$

Pour préserver leur autonomie comme organisations vraiment représentatives de leurs sociétés, les ONG ont l'habitude de ne pas accepter que les représentants de gouvernements en deviennent membres. ${ }^{37}$

Dans le cas de Greenpeace, elle ne demande ni ne sollicite de donations de gouvernements, de corporations ou de partis politiques qui pourraient compromettre son indépendance ou son intégrité. ${ }^{38}$ L'organisation récolte de l'argent surtout par le biais des contributions d'environ 2.8 million de particuliers. ${ }^{39}$

2.2.3. Les ONG sont des entités sans but lucratif: Les ONG n'ont pas comme finalité de faire des profits. Elles ne distribuent pas de bénéfices économiques entre ses membres. "If any profits are earned, they are not and cannot be distributed as such». ${ }^{40}$ Les éventuels profits sont utilisés pour la poursuite de leurs objectifs.

2.2.4. Les ONG sont des entités sans base territoriale: Dans le Droit International Public, seuls les États peuvent occuper et administrer les territoires habitables de la planète. Comme les organisations non gouvernementales n'ont pas de base territoriale, elles sont obligées d'opérer dans un pays donné. Alors, «elles sont soumises aux lois et aux juridictions des autorités de l'État qui les accueille. Une contradiction

décision des campagnes globales de Greenpeace; de développer la présence de Greenpeace dans des régions prioritaires pour l'organisation; etc. L’organisation est gérée par a board of directors composé de sept membres. 35 Greenpeace. About Greenpeace. How is Greenpeace structured. Supra note 34. Chaque filiale est géree par un conseil d'administration, qui désigne un représentant (trustee). Les filiales contribuent avec un pourcentage de leur revenue au budget de Greenpeace International, qui établit les directives principales de l'organisation.

36 Fundamentalprinciples on the status of non-governmentalorganization in Europe and explanatorymemorandum, Council of Europe (13 novembre 2002). Disponible sur <http://www.legislationline.org/legislation.php?tid=2 \&lid $=4971 \&$ less $=$ false $>$.

37 Lindblom, A.-K. Non-governmental organizations in international law. Cambridge: Cambridge University Press, 2005.

38 GreEnPeace. About Greenpeace. Disponible sur <http://www.greenpeace.org/international/about>.

39 Id. Pour poursuivre leurs opérations, ces organisations comptent principalement sur les donations des individus et fondations, principalement. Pourtant, il y a plusieurs organisations qui dépendent de fonds étatiques, ce qui à notre avis a un impact négatif sur leur indépendance.

40 Draft World Bank Handbook on Good Practices Relating to Non-Governmental Organizations (2000), disponible sur <http://www.worldbank.org/>. Par contre, nous ne pouvons pas affirmer que les buts poursuivis par les ONG sont toujours altruistes. Par exemple, certaines ONG se dédient à la promotion commerciale de l'acier, du fer, du pétrole, entre autres. 
émerge ici entre la vocation internationale des ONG, leur raison d'être, et le statut légal dans lequel elles sont inscrites». ${ }^{41}$

2.2.5. Les ONG sont des entités de formes juridiques diverses: Les ONG peuvent adopter différentes formes. Il s'agit communément d'associations mais rien n'empêche qu'elles puissent adopter d'autres formes valables, selon la loi de l'État où elles sont créées. ${ }^{42}$ En fait, Greenpeace International est une dutch stichting une fondation spécifiquement, inscrite dans la Chambre de Commerce des Pays-Bas.

La majorité de ces organisations ont leur siège et la base principale de leurs activités en Europe et aux États-Unis. Le Tiers-monde reste encore mal représenté dans ce type d'institution. ${ }^{43}$ Par exemple, Greenpeace a son siège social à Amsterdam et des filiales au Brésil, en Inde, etc. ${ }^{44}$

2.2.6. Les ONG sont des entités intermédiaires: Sans y être destinées au départ, elles sont devenues les porte-paroles «officieuses» de la société civile. Cette dernière exprime mieux ses craintes et préoccupations mais aussi ses espoirs et défis sur les divers sujets d'actualité à travers les ONG. Nous faisons face ici à une sorte de «rétro-alimentation» parce que les ONG apparaissent comme «le relais privilégié» pour collecter les informations auprès des gouvernements et pour faire remonter vers eux les renseignements recueillis auprès de la société civile. ${ }^{45}$ Les ONG remplissent une fonction éducative vers la population civile, elles interviennent pour la conscientiser quant aux mesures qu'il faut adopter pour la protection de l'environnement. ${ }^{46}$

Le présent travail traite des ONG transnationales, spécifiquement celles qui transcendent les frontières nationales en faisant appel à des buts de solidarité. ${ }^{47}$ Comme nous l'avons mentionné, ce type d'organisations a un siège social principal dans un certain pays et des sections dans d'autres pays.

\footnotetext{
41 MerLe, M. «International non-governmental organizations and their legal status», disponible sur <http:// www.uia.org/legal/app35.php>. Le siège social des ONG conditionne leur soumission à un droit national et implique le fait qu'elles ne soient pas directement appréhendées par le Droit International.

42 En effet, les ONG peuvent aussi adopter la forme de fondations, charités, 'trusts,' comités, etc., selon le droit du pays où le siège social est situé Qu'importe la forme juridique adoptée, les ONG ont besoin d'être garantis de la part des États leur droit d'association, d'assemblée et d'expression afin de pouvoir travailler normalement.

43 Voy. Daillier, P. et A. Pellet. Droit international public. Paris: LGDJ, 2002.

44 Greenpeace. About Greenpeace. How is Greenpeace structured. Supra note 34. Par contre, nous devons noter le rôle essentiel des ONG dans l'aide en faveur des pays du Tiers-monde. Carreau, supra note 22, p. 29.

45 Voy. Lowe, P. «Les ONG et la protection de l'environnement en Afrique Centrale», disponible sur $<$ http:// www.memoireonline.com/03/06/125/m_ong-protection-environnement-afrique-centrale0.html>.

$46 \quad$ Id.

47 La doctrine les appelle aussi, organisations ou associations de solidarité internationale (OSI ou ASI).
} 
La Belgique a promulgué une loi par laquelle elle encourage l'établissement des ONG sur le territoire belge. ${ }^{48}$ En principe, cette loi reconnait aux organisations non gouvernementales ayant leur siège social dans un autre pays, les mêmes droits que ceux reconnus aux entités constituées en Belgique. Cette mesure, unique au monde, permet de conférer des droits à une entité extraterritoriale de droit privé. ${ }^{49}$

Les ONG poursuivent des objectifs divers:

- Aide humanitaire (Associations caritatives, Croix Rouge).

- Coopération pour le développement (OXFAM, Agronomes \& Vétérinaires Sans Frontières).

- Promotion des droits de l'homme (Amnesty International, Human Rights Watch).

- Protection de l'environnement (Greenpeace, Earthwatch Europe, Union Mondiale pour la Nature).

- But politique (Fédérations socialistes, libérales, etc.). ${ }^{50}$

- But social et d'investigation (Associations professionnelles).

\subsection{Différences}

Après avoir expliqué les caractéristiques principales qui définissent les ONG, nous nous attelons dans les lignes qui suivent à indiquer brièvement les différences essentielles qui existent entre ces organisations et d'autres participants non étatiques avec lesquels les ONG transnationales sont souvent confondues.

\footnotetext{
48 Voy. Moniteur, 1er juillet 1921, Loi du 27 juin 1921 relative aux Associations sans But Lucratif, aux Associations Internationales sans But Lucratif et aux Fondations, modifié par Loi promulguée le 2 mai 2002. L'article 58 de cette loi prescrit ainsi: «Les associations internationales poursuivant un but non lucratif d'utilité internationale, valablement constituées à l'étranger conformément a la loi de l'État dont elles relèvent, peuvent ouvrir un siège d'opération. Un siège d'opération est un établissement durable sans personnalité juridique distincte dont les activités sont conformes au but social de l'association internationale poursuivant un but non lucratif d'utilité international [...]». La Convention Européenne sur la Reconnaissance de la Personnalité Juridique des Organisations Internationales non Gouvernementales (24 avril 1986) prévoit aussi une mesure du même caractère que celle de la Belgique, dans son article 2. Malheureusement, jusqu' au 22 août 2006, seulement neuf États européens ont ratifiée cette Convention.

49 Merle, supra note 41. Un des problèmes les plus graves pour les ONG est de savoir comment échapper aux contraintes de la législation de l'État «hôte» et comment gagner la reconnaissance et le respect pour la nature spécifique de leurs activités dans le pays tiers.

50 Lindblom, supra note 37, p. 48. La majorité des instruments internationaux excluent les partis politiques de sa définition. C'est le cas, par exemple, de la Convention Européenne pour la Reconnaissance de la Personnalité Légale des ONG. Les partis politiques sont exclus parce que les buts et activités son centrés sur les problèmes internes d'un pays donné. WIEDERKEHR, M. «La personnalité juridique des organisations nongouvernementales». In: Annuaire Française de Droit International, No XXIII, 1987, p. 750 et s.
} 
2.3.1. Différences avec les sociétés transnationales: Les ONG transnationales sont distinctes des sociétés transnationales car, tandis que ces dernières ont une finalité notamment lucrative, les ONG ne poursuivent aucun but lucratif. ${ }^{51}$ Leur objectif est de tenter d'infléchir ou de corriger l'action des sujets de Droit International, en principe, par une action étendue à minimum deux États (principe repris dans l'article 1 de la Convention Européenne sur la Reconnaissance de la Personnalité Juridique des Organisations Internationales Non Gouvernementales).

2.3.2. Différences avec les organisations internationales: La principale différence que nous pouvons relever entre les ONG et les organisations internationales est que ces dernières possèdent une base intergouvernementale, étant donné que ce sont les États eux-mêmes qui les fondent. Dès lors, les organisations internationales sont appelées sujets de Droit International «dérivés». ${ }^{52}$ En revanche, ces organisations non gouvernementales transnationales sont issues de l'initiative privée, indépendamment d'une quelconque intervention de l'Etat ou d'une organisation internationale.

\subsection{Les organisations non gouvernementales: sujets de Droit International?}

Les États sont sujets de Droit International. Leur souveraineté donne un caractère particulier à leur personnalité internationale. Ainsi, il n'est pas nécessaire de pouvoir invoquer directement la souveraineté pour être reconnu comme sujet de Droit International..$^{53}$ En effet, les sujets de Droit International ne sont pas nécessairement identiques quant à leur nature ou quant à l'étendue de leur droit, leur nature dépend des besoins de la communauté. ${ }^{54} \mathrm{La}$ théorie la plus défendue en doctrine pour attribuer la personnalité internationale à diverses entités est exposée dans les lignes qui suivent.

2.4.1. Les entités ayant des droits et des obligations de caractère international: Le courant majoritaire de la doctrine propose l'attribution de la personnalité internationale à tout sujet ayant des droits et des obligations d'ordre international. Ces entités sont, au premier chef, les destinataires des règles internationales mais ils sont aussi

\footnotetext{
51 Carreau, supra note 22, p. 644. "C'est l'importance de la puissance économique de certaines entreprises multinationales qui commence à changer la conception du droit international classique que leur déniait toute personnalité 'internationale. Ces entreprises, avec l'accord de nombreux États, se sont approprié une certaine personnalité internationale due à la conclusion des contrats avec les États (State contracts) pour l'exploitation des richesses nationales».

52 Id., p. 27. «Le processus de création des organisations internationales a commencé à la fin du XIX siècle (...). Actuellement, il existe plus de 360 organisations internationales de type intergouvernemental et ses actions couvrent tout le spectre des activités humaines, depuis la santé jusqu' au lancement des satellites».

53 Même si selon M. Kelsen le seul sujet du droit international était l'État. DAILlier et as., supra note 43, p. 571.

54 «Reparations for Injuries Suffered in the Service of the United Nations». ICJ Reports 1949, p. 178.
} 
titulaires de droits et obligations résultant du droit des gens. ${ }^{55}$ Néanmoins, seuls les États possèdent une pleine capacité originaire. En revanche, d'autres entités disposent seulement d'une capacité restreinte incluant l'exercice de certains droits et obligations de Droit International qui leur ont été conférées en vertu de leur création. ${ }^{56}$ Le contenu de la personnalité internationale, en termes de capacité, n'est donc pas le même pour tous les sujets de droit. ${ }^{57}$

Cette théorie est issue d'une question posée par l'Assemblée Générale de l'ONU à la Cour International de Justice (ci-après «CIJ»). La question était de savoir si une réparation pouvait être demandée par l'organisation en tant que telle suite au décès de son fonctionnaire, le Comte Folke Bernadotte.

Dans ce cas là, la CIJ a affirmé:

In the opinion of the court, the Organization was intended to exercise and enjoy, and is in fact exercising and enjoying, functions and rights which can only be explained on the basis of possession of a large measure of international personality and the capacity to operate upon an international plane [...] Accordingly, the Court has come to the conclusion that the Organization is an international person ${ }^{58}$

Ainsi, selon cette théorie, la méthode à suivre pour déterminer la personnalité légale internationale d'une entité quelconque, consiste à: «investigating to what extent a particular entity does in fact directly possess some rights, duties and powers (or capacities)». ${ }^{59}$ Des indications intéressantes allant dans ce sens peuvent être remarquées: «la capacité de conclure des traités, d'établir des relations diplomatiques, et de participer aux mécanismes généraux de la responsabilité internationale». ${ }^{60}$

2.4.2. L'accord entre la Suisse et la Fédération Internationale des Sociétés de la CroixRouge et du Croissant-Rouge vis-à-vis de la personnalité internationale des Organisations Non Gouvernementales: Le 29 novembre 1996 a été signé un accord entre la Fédération International des Sociétés de la Croix-Rouge et du Croissant-Rouge (ci-après "Fédération») et le Conseil Fédéral suisse en vue de déterminer le statut juridique de la Fédération internationale en Suisse. ${ }^{61}$ Cet accord n’a pas été le premier

\footnotetext{
55 Voy. Dominice, C. «La personnalité juridique dans le système du droit des gens». In: J. MaKarCRYCK (éd.). Theory of international law at the threshold of the 21 century. La Haye: Kluwer Law International, 1996. 56 Voy. Perez Gonzales, M. «La subjetividad internacional». In: M. DiAz de Velasco. Instituciones de derecho internacional público. Madrid: Tecnos, 2005.

$57 \quad I d .$, p. 258.

58 «Reparations for Injuries Suffered in the Service of the United Nations». ICJ Reports 1949, pp. 178-179.

59 LindBlom, supra note 37 , p. 88.

60 Dominice, supra note 55, pp. 161-163. «Les mécanismes généraux comprennent l’action diplomatique, la présentation de prétentions internationales de manière libre et indépendant de l'existence d'un traité, et même, au besoin la faculté de recourir à des contre-mesures».

61 Gautier, Ph. «ONG et personnalité internationale: A propos de l'accord conclu le 29 Novembre 1996
} 
signé entre la Fédération et un État. En effet, la Fédération depuis 1952 a conclut de nombreux autres accords ayant pour objet de préciser le statut de ses délégations régionales. ${ }^{62}$

Afin de déterminer si cet accord comprend vraiment deux acteurs du Droit International, il faut analyser le texte. Les articles dudit document garantissent la libre disposition de fonds de la Fédération, l'immunité de juridiction et d'exécution. De surcroît, certaines obligations sont imposées à t-elle comme, dans certain cas, renoncer au bénéfice des immunités. ${ }^{63}$ Ainsi que nous pouvons l'observer, ledit accord crée des obligations et des droits internationaux en faveur de la Fédération. Par conséquent, selon la théorie doctrinale majoritaire actuellement, il s'agit d'un nouvel acteur international. ${ }^{64}$

En outre, le texte même de l'accord le mentionne. L'article 1 octroie expressément «la personnalité juridique internationale» à la Fédération. Dans le même sens, l'assimilation des délégations de la Fédération à une représentation régionale d'une organisation internationale — dans plusieurs accords conclus par des États et la Fédération— ${ }^{65}$ est également une autre preuve de la personnalité juridique internationale de la Fédération. Pourtant, même si la Fédération est à l'origine une association, sa condition, son statut dans le Droit International ne peut pas être directement transposable aux autres ONG.

La Fédération a atteint ce statut grâce à son apport au Droit International Humanitaire depuis sa création, c'est un cas sui generis au point qu'elle a acquis le statut d'observateur auprès de l'Assemblée Général des Nations Unies. ${ }^{66}$ Donc, toutes les

entre la Suisse et la Fédération International des Sociétés de la Croix-Rouge et du Croissant-Rouge». In:Revue Belge de Droit International, № 1, 1997, p. 172 et s. La Fédération est créée en 1919 sous le nom de Ligue des Sociétés de la Croix-Rouge, grâce à l'initiative des Sociétés de la Croix-Rouge des États-Unis, de Royaume-Uni, etc. Son objectif est de faire progresser l'action humanitaire des Sociétés nationales, en vue de prévenir et d'alléger les souffrances humaines. La Fédération a une personnalité juridique distincte du Comité International de la Croix Rouge (C.IC.R) «La Fédération est responsable de l'organisation des secours en cas de catastrophes naturelles et technologiques; le C.I.C.R. est responsable de l'assistance en faveur des conflits armes. En cas de conflits, la Fédération joue également un rôle car elle assiste les victimes en dehors de la zone de conflit et peut également agir dans la zone de conflit en coopération avec le C.I.C.R. et sous sa direction». D'ailleurs, la Fédération représente officiellement les Sociétés Nationales de la Croix Rouge au niveau international.

${ }_{62} I d$., p. 175. Le but de ces accords est de garantir une certaine autonomie dans l'ordre local dans le quel est insérée.

${ }_{63}$ Id., p. 176-177

64 Le seul fait de signer un traité international avec un État est un indicateur que nous sommes devant un sujet de Droit International parce que cette attribution est réservée aux sujets ayant une personnalité internationale.

65 Gautier, supra note 61, p. 184. Cette assimilation se produit en cas de libertés de communication, par exemple. «Dans le même sens, plusieurs accords disposent que le règlement des matières non prévues par ceux-ci, sera effectué conformément aux principes généraux de droit international et/ou la Convention de Vienne de 1961 sur les relations diplomatiques».

66 En fait, la résolution de l'Assemblée de Nations Unies 49/2 de 1994 par la quelle l'octroie le statut 
ONG transnationales ne sont pas sujets de Droit International et ne peuvent pas arriver à avoir une telle condition.

Actuellement, les ONG transnationales sont considérées comme des associations de droit interne et relèvent du droit national du lieu d'établissement de leur siège social. ${ }^{67}$ "Non governmental organizations do not have international legal personality and are therefore incapable of participating directly in the customary process» ${ }^{68}$. De plus, l'instinct de conservation fait que les États sont peu disposés à octroyer la personnalité juridique à des organisations lorsque cela peut porter préjudice à leur propre autorité. $^{69}$

Ainsi, nous devons procéder à une analyse cas par cas pour déterminer si telle ONG transnational a la condition de sujet de Droit International. «L'octroi d'une telle qualité repose sur un critère fonctionnel. L'organisation qui en bénéfice devant démontrer une certaine filiation étatique, que ce soit sous l'angle de ses membres ou de ses activités» ${ }^{70}$. Or, l'octroi a priori de la qualité du sujet de droit de gens aux ONG transnationales n'est pas envisageable, actuellement.

\section{Les Organisations Non Gouvernementales et leurs statuts dans le Droit International de l'Environnement}

Selon Monsieur Carreau, les ONG sont essentiellement des groupes de pression internationaux. ${ }^{71}$ Nous sommes partiellement d'accord avec cette affirmation dans le

d'observateur reconnait le rôle spécial joué par cette association dans ce domaine, quand t-elle mentionne: «Recalling the special functions of the member societies of the International Federation of Red Cross and Red Crescent Societies which are recognized by their respective governments as auxiliaries to the public authorities in the humanitarian field on the basis of the Geneva Conventions of 12 august 1949». Cependant, apparemment ce statut d'observateur ne pourrait pas être acquis par une autre ONG parce que le 9 décembre 1994, l'Assemblée Générale a pris une décision au terme de laquelle «the granting of observer status in the General Assembly should in the future be confined to States and to those intergovernmental organizations whose activities cover matters of interest to the Assembly». Cette résolution exclue donc «non governmental organizations». GAUTIER, supra note 61, p. 181.

67 Voy. Lowe, P., supra note 45.

68 Voy. Byers, M. Custom, power and the power of rules: International relations and customary international law. Cambridge: Cambridge University Press, 1996, cité dans Lindblom, supra note 37, p. 105. Ceux qui s'opposent à octroyer de personnalité internationale aux ONG disent en plus: «NGOs form a genuine nebula including both giants and dwarfs. But the differences in size and power are not the only ones. To grant them all the same status and privileges (conferring exemptions from the ordinary law, particularly with regard to taxation, customs, labor regulations, etc.) would be tantamount to giving identical treatment to a learned or charitable society and to the directors of a multinational company or consortium». MerLE, supra note 41.

69 MerLe, supra note 41.

70 Gautier, supra note 61, p. 188. «Par ailleurs, faire parti des acteurs de droit international requiert une invitation. C'est une affaire de reconnaissance par la communauté internationale».

71 Carreau, supra note 22, p. 29. 
sens où les ONG remplissent cette fonction depuis leurs origines. Ceci étant, avec les cours du temps, les ONG ont acquis d'autres fonctions plus importantes (voyez infra point 2.1 et suivants).

Vu la transcendance des ONG comme groupes de pression, nous dirons que grâce à leurs actions directes non violentes et courageuses sacrifiant leur propre intégrité; elles ont obtenu des résultats d'une importance capitale. Par exemple, suite aux actions de Greenpeace les États-Unis ont arrêté leur campagne d'essais nucléaires à Amchitka, en $1971 .{ }^{72}$ En 1985, les essais nucléaires ont à nouveau fait l'objet d'une controverse internationale, après le sabotage par les services secrets français du Rainbow Warrior, navire amiral de Greenpeace. ${ }^{73}$ Ce dernier essayait d'arrêter pacifiquement les essais nucléaires français.

De même, les ONG constituent des groupes de pression parce que, de manière quotidienne, elles attirent l'attention du grand public sur des problèmes environnementaux, cherchant à informer et sensibiliser la société civile. Cela est dû au fait que la sensibilisation est une tâche cruciale pour les ONG ${ }^{74}$ Plusieurs moyens dont les médias peuvent être utilisés à cette fin (publication de périodiques, tenue de séminaires et d'ateliers de formation, etc.). ${ }^{75}$

Lautre forme d'action des ONG comme groupe de pression est le lobbying; c'est-àdire l'activité d'essayer d'influencer des législateurs ou d'autres fonctionnaires publiques avec pouvoir de décision. ${ }^{76}$

What is undeniable $[\ldots]$ is that environmentalists $[\ldots]$ have forced their way into the previously closed rooms of international diplomacy. Even if their policies are not adopted, they are there, placing their position papers on the table and speaking out, not just in the corridors but in the once sacrosanct plenary halls and in the small, out of the way chambers where deals are hammered out in the secret meetings. ${ }^{77}$

Ainsi, par exemple, grâce au lobbying exercé pendant de nombreuses années par Greenpeace et d'autres $\mathrm{ONG}$, les gouvernements du monde entier ont signéla première

\footnotetext{
72 Froeur, N. «Le positionnement distancié de Greenpeace». In: Revue Française de Sciences Politiques, № 54, 2004, p. 421 et s., cité dans Soumy, supra note 10.

73 GreENPEACE, supra note 29.

74 Lowe, supra note 45. "Ces mesures sont d'autant plus importantes que le public constitue un moyen de pression puissant sur les autorités étatiques». 75 Id.

$76 I d$., «[...] on doit dire que le pouvoir d'information et de sensibilisation des décideurs par les ONG fait logiquement suite à l'obligation qui pèse sur les États de mettre à la disposition des citoyens les informations relatives à l'environnement qu'ils détiennent».

77 Shabecoff, P. A New Name for Peace: International Environmentalism, Sustainable Development, and Democracy. Hanover, NH: University Press of New England, 1996, cité dans D. Hunter, J. Salzman et D. Zaelke. International Environmental Law and Policy. New York: Foundation Press, 2002.
} 
Convention de Stockholm sur l'Élimination des Polluants Organiques Persistants en $2001 .^{78}$ De même, l'application effective de la loi sur les crimes environnementaux au Brésil depuis 1999 a été obtenue suite aux pressions exercées par Greenpeace. ${ }^{79}$ Ainsi, «NGOs have played a crucial role in setting the international agenda, in influencing international rule-making and in contributing to the implementation of international norms. They have proven to be a driving force in some of the major innovations undergone in the international system...». ${ }^{80}$

\subsection{Les organisations non gouvernementales devant les juridictions internationales}

«L'obligation générale qu’ont les États de veiller à ce que les activités exercées dans les limites de leur juridiction ou sous leur contrôle respectent l'environnement dans d'autres États ou dans des zones ne relevant d'aucune juridiction nationale fait maintenant partie du corps de règles du droit international de l'environnement». ${ }^{81} \mathrm{Cer}-$ taines juridictions internationales traitent déjà des affaires concernant ce domaine de la protection de l'environnement si délicat et transcendental pour la propre conservation de l'espèce humaine.

3.1.1. La Cour International de Justice: A ce jour, il n’existe pas encore un véritable mécanisme juridictionnel international propre en droit de l'environnement. ${ }^{82}$ Ceci étant, la Cour Internationale de Justice, organe judiciaire principal des Nations Unies, est compétente pour connaître toute sorte de différends interétatiques tels que, par exemple, les problèmes environnementaux. ${ }^{83}$ A cet effet, a été créée en 1993 une Chambre pour les questions d'environnement, composée de sept membres. ${ }^{84}$

\footnotetext{
78 GREENPEACE, supra note 29. Ces polluants sont classés "parmi les produits chimiques les plus dangereux connus et qui, par leur accumulation dans l'environnement, constituent une menace sérieuse pour la santé humaine». Guruswamy, L. International Environmental Law. Saint Paul: Thomson West, 2003. Grâce à son travail, Greenpeace a été invitée à participer comme observateur dans les négociations de cette Convention.

79 GreEnpeace, supra note 29. Depuis 1999, «les montants des amendes, à la charge des sociétés utilisant des bois coupés illégalement en Amazonie, sont augmentés de manière substantielle».

80 Voy. Rebasti, E. et L. Vierucci. «Legal Status for NGOs in Contemporary International Law?», disponible sur <http://www.esil-sedi.eu/english/pdf/VierucciRebasti.PDF>. «It is well-known that actio popularis is virtually unknown in international law, with the only and limited exception of the Inter-American and African Commission on Human Rights and, to some extent, the African Court on Human and People's Rights».

81 Licéité de la menace ou de l'emploi d'armes nucléaires, supra note 8.

82 Breton-Legoff, supra note 4.

83 Daillier et as., supra note 43, p. 894

84 Voy. Ranjeva, R. «L'environnement, la Cour Internationale de Justice et sa chambre spéciale pour les questions d'environnement». In: Annuaire Français de Droit International, N XL, 1994, p. 433 et s. La procédure contentieuse relative au Projet Gabcikovo-Nagymaros (Hongrie-Slovaquie) et les demandes d'avis sur les armes nucléaires présentés par l'Assemblée de l'Organisation Mondiale de la Santé et l'Assemblée Générale des Nations Unies ont posé des questions importantes sur le droit international en matière d'environnement.
} 
Après une certaine incertitude sur la pratique de la CIJ quant au rôle des ONG devant la Cour, celle-ci a émis en 2004 l'instruction de procédure XII. Cette mesure fait suite à la déclaration suivante de l'ancien juge Kooijmans de la CIJ. «The Court is increasingly confronted with issues which are not strictly of inter-party relevance and do not have merely effect on bilateral relations between States [...] the Court seems to recognize the global values which are invoked by non-State actors $[\ldots] » .^{85}$ Cette règle de procédure établit:

1. Lorsqu'une organisation non gouvernementale présente, de sa propre initiative, un exposé écrit et/ou un document dans le cadre d'une procédure consultative, cet exposé et/ou ce document ne doivent pas être considérés comme faisant partie du dossier de l'affaire.

2. Pareils exposés écrits et/ou documents sont traités comme des publications facilement accessibles, et les États et les organisations intergouvernementales présentant des exposés écrits ou oraux en l'affaire concernée peuvent s'y référer au même titre qu'aux publications relevant du domaine public. ${ }^{86}$

Pourtant, cette procédure n'est pas invoquée très souvent dans la pratique de la CIJ. ${ }^{87}$

Néanmoins, les ONG tendent — parfois— à agir à «'abri des projecteurs». ${ }^{88} \mathrm{Un}$ exemple célèbre est la campagne des O.N.G. qui a mené aux avis consultatifs sur

Cela est également le cas de l'affaire entre l'Uruguay et l'Argentine sur «las papeleras» en débat actuellement. Certains auteurs sont en faveur d'une cour internationale de l'environnement. La question se pose de savoir si serait vraiment nécessaire. Pour quelques commentaires à ce sujet, voy. R. RANJEVA, «Les potentialités des modes juridictionnels internationaux des règlements des différends». In: C. ImPERIALI (éd.). «L'effectivité du droit international de l'environnement. Paris: Economica, 1998.

85 Kooijmans, P. «The role of non-state actors and international dispute settlement». In: W. P. Heere, From Government to Governance: The Growing Impact of Non-state Actors on the International and European Legal System, 2004, cité dans Rebasti et as., supra note 80.

86 Voy. Cour International de Justice, Documents de base, Textes constitutifs, Instructions de procédure, disponible sur <http://www.icj-cij.org/cijwww/cdocumentbase/cbasictext/fCour_Instructions-procedures.pdf>.

87 Voy. Currie, D. «The experience of Greenpeace International». In: T. Treves et as. (éd.). Civil Society, International Courts and Compliance Bodies. La Haye: T.M.C. Asser Press, 2005.

88 «Practice shows that formal restrictions to non-governmental participation do not prevent NGOs from participating informally and «beyond the rules», for example by joining «umbrella organizations» which already enjoy the consultative status or simply by continuing the practice of informal consultations with the Intergovernmental Organizations». MerLe, supra note 41. Tout à fait, les ONG cherchent toujours les moyens pour atteindre leurs buts. Ainsi, en profitant du fait que certains règlements de procédure permettent aux victimes de se faire représenter à l'instance par des juristes de leur choix; il est arrivé assez fréquemment que des ONG représentent des victimes devant la Cour Européenne de Droits de l'Homme ou les conseillent sans les représenter. Mais, les ONG peuvent servir de conseil aux même États devant des juridictions internationales comme dans le cas des avis consultatifs, comme nous sommes en train d'analyser. BRETON-LEGOFF, supra note 4. 
les armes nucléaires. ${ }^{89}$ Le projet a été soutenu en 1992 par trois ONG: International Physicians for the Prevention of Nuclear War. International Peace Bureau et International Association of Lawyers against Nuclear Arms. Il cherchait à influencer les pays membres de l'Organisation Mondiale de la Santé et de l'Assemblée Générale de l'ONU pour passer des résolutions, demandant des avis consultatifs sur la licéité de la menace ou de l'emploi d'armes nucléaires. Naturellement, l'idée était que la COJ devait déclarer l'emploi d'armes nucléaires complètement illégal. ${ }^{90}$

En réalité, la participation des ONG dans les affaires portant sur la légalité des essais nucléaires devant la Cour semble être allé plus loin que le lobbying: les ONG auraient non seulement rédigé les mémoires de certains pays devant la CIJ mais elles «auraient également été incluses dans la délégation étatique». ${ }^{91}$ Malheureusement, nous connaissons déjà les réponses données par la $\mathrm{CIJ}$ dans les deux questions proposées sous forme d'avis consultatifs. ${ }^{92}$

Le juge Guillaume de la CIJ a décrit la résolution de l'Assemblée Générale des Nations Unies comme originaire dans l'activité des ONG. Dans son opinion, il a affirmé:

I am sure that the pressure brought to bear in this way did not influence the Court's deliberations, but I wondered whether, in such circumstances, the request for opinions could still be regarded as coming from the Assemblies which had adopted them or whether, piercing the veil, the Court should not have dismissed them as inadmissible. However, I dare to hope that Governments and intergovernmental institutions still retain sufficient independence of decision to resist the powerful pressure groups which besiege them today with the support of mass media $\{\ldots\}^{93}$

Evidemment, le juge Guillaume n'était pas en faveur de l'implication de la société civile dans le plan internationale. En revanche, la juge Higgins a affirmé sur ce sujet,

\footnotetext{
89 Voy. SANDS, P. «International Law, the practitioner and non-state actors». In: C. WICKREMASINGHE (éd.). The International Lawyer as Practitioner. London: The British Institute of International and Comparative Law 2000, cité dans LindBLom, supra note 37, p. 219.

90 Voy P. NANDA et as., «Nuclear weapons and the world court», Transnational Publishers 1998, cité dans Lindilom, supra note 37, pp. 219-221. «The World Court Project obtained the support of more than 700 groups of civil society. Through these groups, the Project gathered Declarations of Public Conscience, in which individuals declared their oppositions toward nuclear weapons. [...] At the outset of the oral hearings at the ICJ in October 1995, more than 3.5 million Declarations of Public Conscience were presented to the Court».

91 Breton-Legoff, supra note 4.

92 Les avis consultatifs auxquels nous nous référons sont: l'avis consultatif sur la Licéité de la Menace ou de l'Emploi d'Armes Nucléaires du 8 juillet 1996, demandé par l'Organisation Mondiale de Santé et celui à propos de la Licéité de l'Utilisation des Armes Nucléaires par un État dans un conflit armé, demandé par l'Assemblée Générale des Nations Unies.

93 Legality of the Use by a State of Nuclear Weapons in Armed Conflict, Separate Opinion of Judge Guillaume, ICJ Reports 1996, pp. 287-288.
} 
«clearly to some, these radical phenomena represent the democratization of international law». ${ }^{94}$

3.1.2. Le Tribunal International du Droit de la Mer: De leur côté, les États ont crée des juridictions plus spécialisées qui ne peuvent connaître que de certains types de litiges. ${ }^{95}$ Le Tribunal International du Droit de la Mer a pour mission de connaître des différends relatifs à l'interprétation ou à l'application de la Convention des Nations Unies sur le droit de la mer ${ }^{96}$ ainsi que des questions expressément prévues dans tout autre accord conférant compétence au Tribunal. ${ }^{97}$ En 1997, ont été constituées trois chambres spéciales auxquelles peut être soumise une affaire, à la demande des parties du différend. Parmi ces chambres figure la Chambre de l'environnement marin. ${ }^{98}$

Cependant, les ONG ne peuvent pas intervenir comme parties demanderesses devant le Tribunal. Jusqu'au présent, ces organisations n'ont pas non plus cherché à participer propio motu comme amicus curiae ni à soumettre des informations non sollicitées dans des procédures devant le Tribunal. ${ }^{99}$ Ceci étant, "given the fact that the cases dealt with by the Tribunal often concerned issues relating to the protection of the marine environment, a subject matter with respect to which NGOs are particularly active, this issue is likely to arise in the future». ${ }^{100}$ Nous espérons que lorsque cela arrivera, la Chambre de l'Environnement Marin adoptera une interprétation souple de ses règles de procédure, en favorisant la participation des ONG.

3.1.3. Les juridictions régionales: Au niveau régional, la Cour Africaine des Droits de l'Homme et des Peuples ${ }^{101}$ «ne pourra être saisie par les personnes privées ou les ONG que si l'État mis en cause a accepté cette procédure (art. 5 et 34, \$6, du Protocole

94 Higgins, R. «The reformation in international law». In: Lindblom, supra note 37, p. 223.

95 DAILliER et as., supra note 43, p. 911.

96 Id., p. 912. La Convention de «Montego Bay» de 1982 régit l'ensemble des questions juridiques concernant les espaces maritimes et leurs ressources (pêche, pollution, délimitation, etc.).

$97 \quad$ Id., p. 913.

98 Voy. Tribunal International du Droit de la Mer, Actualité, Communiqués de presse ITLOS/Press 5, 3 mars 1997, disponible sur <http://www.itlos.org/start2_fr.html >.

99 Voy. Gautier, Ph. «NGOs and Law of the Sea Disputes». In: Treves et as. (éd), supra note 87, p. 242. Currie, supra note 87, p. 161. "The Statute of the Tribunal (of the Sea) is silent on the receipt of amicus curiae briefs as such. Article 31 of the Statute provides for intervention by additional states upon request to the Tribunal. Article 84 of the Rules provide that ITLOS may at, any time prior to the closure of the oral proceeding, at the request of a party or propio motu, request and appropriate intergovernmental organization to furnish information relevant to a case before it. ITLOS has discretion in regulating its procedure [...]».

100 Gautier, supra note 99.

101 Voy. La Fédération Internationale des Ligues des Droits de 1 'Homme, 10 clés pour comprendre et utiliser la Cour Africaine des Droits de l'Homme et des Peuples, disponible sur <http://www.fidh.org/rubrique. php3?id_rubrique=346>. Depuis le 25 janvier 2004, est entrée en vigueur le Protocole créant la Cour Africaine des Droits de l'Homme et des Peuples. Ainsi, l'Afrique compte un nouvel organe de protection des droits de l'homme qui complémente le rôle de la Commission Africaine des Droits de l'Homme et des Peuples. Une des différences fondamentales de la Cour Africaine par rapport à la Commission, qui examine les mêmes types 
Relatif a la Charte Africaine des Droits de l'Homme et des Peuples portant Création d'une Cour Africaine des Droits de l'Homme et des Peuples)». ${ }^{102}$

Au niveau européen, la Cour Européenne des Droits de l'Homme (ci-après CEDH) est une juridiction dont la mission spécifique consiste à contrôler le respect des droits de l'homme en Europe, consacrés par la Convention de Sauvegarde des Droits de l'Homme et des Libertés Fondamentales. Depuis l'entrée en vigueur des protocoles $\mathrm{n}^{\circ} 9$ et 11 en 1998, la CEDH peut être saisie par tout individu, toute ONG ou tout groupe de personnes qui se considère victime de la violation d'un droit reconnu par la convention. ${ }^{103}$ Bien que le droit à l'environnement sain ne soit pas expressément protégé par la Convention, des allégations concernant les incidences négatives de certaines pollutions sur le droit à la vie (art. 2 de la Convention) et le respect de la vie privée et familiale (art. 8 de la Convention) ont été exprimés devant la CEDH pour obtenir sa protection. ${ }^{104}$

Quant à la Cour de Justice des Communautés Européennes, elle assure le respect du droit dans l'interprétation et application du traité. Cette mesure a été interprétée comme concernant également l'ensemble du droit communautaire «dérivé», dont on connaît l'ampleur dans tous les domaines où existent des politiques communes, ${ }^{105} \mathrm{en}$ ce compris les politiques environnementales. ${ }^{106}$ Dans l'arrêt de la Cour de Justice des

de violations des droits de l'homme, est la judiciarisation de la procédure d'examen des requêtes. Les décisions de la Cour Africaine ont force obligatoire, contrairement aux décisions de la Commission.

102 Daillier et as., supra note 43, p. 695.

103 Id., p. 916. Bien que la CEDH ne prévoie pas l'institution d'actio popularis, elle permet l'intervention des ONG comme amicus curiae. Frigessi, M. «NGOs before the european court of human rights». In: Treves et as. (éd.), supra note 87, p. 58. Par rapport au nombre des affaires jugées par la $\mathrm{CEDH}$, les cas où les allégations des ONG comme amicus curiae ont été acceptés sont très rares. LindBlom, supra note 37, p. 364.

104 Daillier et as., supra note 43, p. 1272. En se référant aux arrêts Oneryiliz c. Turquie et Hatton c. Royaume-Uni, nous pouvons constater que le problème se centre sur le fait que les requérants —-même les ONG - doivent prouver, d'une part, qu'ils ont été personnellement et directement victimes d'une violation des droits prévus par la Convention ou ses protocoles et, d'autre part, que la violation a été commise par l'un des États liés par la Convention. Cette question est remplie, sans problème, si elles peuvent alléguer l'existence d'une atteinte à leur santé ou à leur vie, elle est plus délicate à remplir lorsqu' une atteinte à la qualité de vie en général est alléguée. Dans cette hypothèse, le préjudice est moins direct et plus difficilement démontrable. La difficulté de prouver les dommages environnementaux se présente donc comme un obstacle.

105 Id., p. 921.

106 Voy. Europa Le Portail de l'Union Européenne, Press room, Press releases, 10 janvier 2007, disponible sur $<$ http://europa.eu/rapid/pressReleasesAction.do?reference=IP/07/29\&format=HTML\&aged=0\&language= FR\&guiLanguage=en>. Les institutions communautaires sont compétentes pour fixer des politiques communes pour les États membres en matière d'environnement. Au propos, la Commission vient récemment de «[... propose(r) [...] un paquet global de mesures établissant une nouvelle politique énergétique pour l'Europe afin de lutter contre le changement climatique et de renforcer la sécurité énergétique et la compétitivité de l'UE. [...] La Commission est convaincue que la conclusion d'un accord international sur le cadre post2012 devrait entraîner, pour 2020, une réduction de 30\% des émissions produites par les pays développés. Pour marquer encore davantage son engagement, la Commission propose que l'Union Européenne s'engage 
Communautés Européennes (ci-après «CJCE») «Greenpeace International c. Commission Européenne» a été analysée la problématique du locus standi des ONG. ${ }^{107}$

3.1.4. Greenpeace International c. Commission Européenne: Cette affaire concerne la décision C (91) 440 prise par la Commission en 1991, accordant au Royaume d'Espagne une assistance financière pour la construction de deux centrales électriques dans les îles Canaries par l'Unión Eléctrica de Canarias SA (ci-après «Unelco»). ${ }^{108}$

En cette affaire, deux des dix-neuf requérants (parmi eux, des personnes physiques et des associations), ont informé la Commission que l'Unelco avait omis d'effectuer une étude d'incidences conformément à ce qu'exigeait la directive 85/337 du Conseil. Les requérants ont demandé d'intervenir au Conseil afin que les travaux soient arrêtés. ${ }^{109}$

Sans trouver réponse à ces demandes, les requérants sont allés devant le Tribunal de Première Instance, afin d'obtenir l'annulation de ladite décision de la Commission. ${ }^{110} \mathrm{La}$ Commission a soulevé une exception d'irrecevabilité à l'appui de laquelle elle faisait valoir deux moyens, l'un tiré de la nature de l'acte attaqué et l'autre du défaut de qualité pour agir des requérants. Le Tribunal a accueilli l'exception. ${ }^{111}$

Par requête déposée le 16 octobre 1995, Greenpeace International et les autres requérants ont formé un pourvoi contre l'ordonnance du Tribunal de Première Instance cité, en tant qu'il avait déclaré irrecevable leur recours. ${ }^{12}$ Après examen attentif, la Cour a décidé que l'interprétation de l’article 230, \$4, C.E., ${ }^{113}$ retenue par le Tribunal

maintenant à réduire d'au moins $20 \%$ ses émissions de gaz à effet de serre d'ici 2020, par le biais notamment de mesures dans le domaine de l'énergie».

107 Dupuy, P.M. «L'unité de l'ordre juridique international». In: RCADI, No 297, 2002, p. 114 et s. Le locus standi serait un «droit d'action en justice». LindBLom, supra note 37, p. 364. La CJCE emploie une pratique restrictive par rapport à l'amicus curiae, en requérant que l'intervenant ait un intérêt direct et concret dans le cas.

108 Voy. Cour de Justice des Communautés Européennes, 2 avril 1998, Greenpeace International c. Commission Européenne, $\mathbb{\$}$ 2, alinéa 1-2, disponible sur <http://curia.europa.eu/jurisp/cgi-in/form.pl?lang=fr\& newform=newform \&Submit=Rechercher\&alljur=alljur\&jurcdj=jurcdj\&jurtpi=jurtpi\&jurtfp=jurtfp\&alldoc $\mathrm{rec}=$ alldocrec $\&$ docj $=$ docj\&docor $=$ docor $\&$ docop $=$ docop $\&$ docav $=$ docav $\&$ docsom $=$ docsom $\&$ docinf $=$ docinf $\&$ alldocnorec=alldocnorec\&docnoj=docnoj\&docnoor $=$ docnoor\&typeord $=$ ALLTYP\&allcommjo=allcommjo \&affint $=$ affint $\&$ affclose $=$ affclose $\&$ numaff $=\&$ ddatefs $=\&$ mdatefs $=\& y d a t e f s=\&$ ddatefe $=\&$ mdatefe $=\& y$ datefe $=$ $\&$ nomusuel=greenpeace $\&$ domaine $=\&$ mots $=\&$ resmax $=100>$. Selon l'article 5 de ladite décision, la Commis sion pourrait réduire ou suspendre l'aide apportée à l'opération en cause, si l'examen de cette dernière révélait l'existence d'irrégularités.

$109 I d ., \$ 2$, alinéa 3 .

$110 \quad I d ., \$ 3$.

$111 \quad I d ., \$ 4-5$.

$112 \quad I d ., \$ 1$.

113 Art. 230 du Traité de la Communauté Européenne, «Toute personne physique ou morale peut former, dans les mêmes conditions, un recours contre les décisions dont elle est le destinataire et contre les décisions qui, bien que prises sous l'apparence d'un règlement ou d'une décision adressée à une autre personne, la concernent directement et individuellement». 
pour conclure au défaut de qualité pour agir des requérants, est conforme à la jurisprudence constante de la Cour qu'exige que les requérants soient directement et individuellement concernés. ${ }^{114}$

De la sorte, au sujet de locus standi des associations comme Greenpeace et d'autres associations requérantes, le Tribunal a rappelé qu'une association constituée pour promouvoir les intérêts collectifs d'une catégorie de justiciables ne saurait être considérée comme étant individuellement concernée par la dite décision du Conseil et, par conséquent, n'est pas recevable à introduire un recours en annulation lorsque ses membres ne sauraient le faire à titre individuel. ${ }^{115}$ La CJCE a confirmé que Greenpeace «ne justifiait pas d'un intérêt à agir pour invoquer le non-respect par un État membre d'une décision adressée à celui-ci en matière d'environnement». ${ }^{116} \mathrm{La}$ CJCE a donc rejeté le pourvoi le 2 avril 1998. ${ }^{117}$

Quelques auteurs soutiennent que le refus de la Cour de réviser sa jurisprudence résulte de questions procédurales, spécifiquement, la peur d'être «inondée» de pourvois. ${ }^{118}$ Nous sommes de l'opinion qu'avec ce type de arrêts, la CJCE parait prêt à accepter la commission d'infractions contre ladite décision du Conseil en sachant qu' «il existe des actes normatifs à caractère général qui ne toucheront aucune personne de façon personnelle et directe. En cas d'inégalité de tels actes, aucune personne ne pourrait recourir en justice $[\ldots] » .{ }^{119}$

Justement, en sachant le caractère éminemment collectif (au lieu d'individuel) des intérêts environnementaux; ${ }^{120}$ une interprétation plus large de l'article 230 C.E. devrait être prise par la CJCE ou, en tout cas, une modification du texte actuel dudit article doit être entamée. ${ }^{121}$

\footnotetext{
114 Cour de Justice des Communautés Européennes, supra note 108, $\$ 27$.

$115 \quad$ Id., $\$ 14$.

116 DaILlier et as., supra note 43, p. 1281.

117 Lindilom, supra note 37, p. 267-268.

118 Id., p. 268.

119 AREND, E. «Luxembourg: La longue marche des associations de défense de l'environnement au prétoire».

In: Revue Européenne de Droit de l'Environnement, No 2, 1998, p. 164 et s.

120 Gerard, N. «Access to the European Court of Justice: A lost opportunity». In: Journal of Environmental Law, $\mathrm{N}^{\circ} 10,1998$, p. 331 et s.

121 ZilleR, J. «Les instruments juridiques de la protection des intérêts diffus et des biens collectifs: Le rôle des pouvoirs privés et la rentrée des pouvoirs publics». In: Revue Internationale de Droit Économique, $\mathrm{N}^{\circ} 3-4$, 2003, disponible sur <http://www.cairn.info/article.php?ID_REVUE=RIDE\&ID_NUMPUBLIE=RIDE_ 173\&ID_ARTICLE=RIDE_173_0495>. M. Ziller fais bien en annoter que dans le projet de Constitution Européenne, la protection des intérêts diffus est renforcée via l'article III-365, $\$ 4$. Le texte proposé va audelà de l'article 230 C.E. en vigueur. «Toute personne physique ou morale peut former, dans les conditions prévus aux paragraphes 1 et 2 , un recours contre les actes dont elle est le destinataire ou qui la concernent directement et individuellement, ainsi que contre les actes réglementaires qui la concernent directement et qui ne comportent pas de mesures d'exécution». «Quant aux mesures à caractère général (c'est la signification
} 
Ce qui est clair ce que la $\mathrm{CJCE}$ a laissé passer l'opportunité de rappeler à toute l'Union que les actes communautaires en matière environnementale -où il existe beaucoup des problèmes d'inobservance de normes de la part des États membresont aussi un caractère contraignant. ${ }^{122}$

\subsection{Les organisations non gouvernementales face aux mécanismes quasi juridictionnels internationaux}

Certaines entités ne peuvent être qualifiées de «juridictionnelles» parce que ce ne sont pas des tribunaux à proprement parler. Ainsi, si ces organismes agissent comme les tribunaux, ils ne rendent pas de décisions ayant un caractère obligatoire. ${ }^{123}$ Parmi ces entités se trouve la Commission Africaine des Droits de l'Homme et des Peuples (ciaprès "Commission») qui a comme fonction principal d'examiner les communications introduites par les individus, les ONG et les États parties à la Charte Africaine, alléguant des violations des droits de l'homme par ces États. ${ }^{124}$

Cette Commission est qualifiée de quasi juridiction internationale parce que lorsque la Commission constate que les violations ont eu lieu, elle a seulement le pouvoir de faire des recommandations à l'État visé, afin qu'il s'assure qu'une enquête soit menée sur ces allégations, et que des mesures soient prises pour éviter que cela ne se reproduise. ${ }^{125}$

3.2.1 L'Actio Popularis: Avant d'analyser la recommandation faite par la Commission au Gouvernement de Nigeria à propos du peuple d'Ogoniland, il importe de préciser ce qu'est l'actio popularis. Il est le mécanisme procédural ayant pour objet de défendre un intérêt collectif devant une juridiction internationale. Ainsi, la Charte Africaine permet aux ONG d'exercer cette action et d'entamer une procédure judiciaire devant la Commission pour la violation de droits de l'homme. ${ }^{126}$

3.2.2. Social and Economic Rights Action Center, Center for Economic and Social Rights c. Nigeria: Au Nigeria, le delta du Niger comporte de vastes ressources de

du terme « réglementaire " dans la théorie juridique dans la plupart des États membres) ne comportant pas de mesures d'exécution, elles pourront être contestées directement par la voie du recours en annulation par toute personne intéressée. Les personnes cherchant à protéger les intérêts diffus [...] disposeront dès lors d'une panoplie complète de recours contre les mesures pertinentes et qu'elles estimeraient contraires à la constitution".

122 Gerard, supra note 120, p. 345.

123 Les décisions de ce type d'entités sont plutôt de caractère moral.

124 Commission Africaine des Droits de l'Homme et des Peuples. A propos de nous, Mandat. Disponible sur $<$ http://www.achpr.org/francais/_info/mandate_fr.html>.

125 Charte Africaine des Droits de l'Homme et des Peuples, supra note 20, article 46.

126 ReBAsti et as., supra note 80. «It is well-known that actio popularis is virtually unknown in international law, with the only and limited exception of the Inter-American and African Commission on Human Rights and, to some extent, the African Court on Human and People's Rights». 
pétrole. Cette région inclut le people d'Ogoni qui y habite la depuis plus de 500 ans. ${ }^{127}$ Plusieurs compagnies ont commencé des opérations pour l'exploration et l'extraction pétrolière dans les années cinquantes. De 1970 à 1982, 1581 incidents de renversement de pétrole ont été effectués dans le delta de la rivière Niger. Le 14 mars 1996, deux organisations non gouvernementales, l'une des États-Unis et l'autre de Nigeria ont, en tant que représentants du peuple d'Ogoniland, présenté une communication contre le gouvernement de Nigeria devant la Commission. ${ }^{128}$

Les demandeurs allégeaient que le gouvernement de Nigeria ${ }^{129}$ était directement impliqué dans la production du pétrole via la Nigerian National Petroleum Company (ci-après «NNPC») associée dans un consortium avec Shell Petroleum Development Corporation (ci-après «SPDC»). Leurs opérations —allèguent les $\mathrm{ONG}$ — ont causé une dégradation environnementale et des problèmes de santé pour le peuple d'Ogoniland. ${ }^{130}$ D'ailleurs, la communication alléguait une violation concertée d'un grand éventail de droits garantis au titre de la Charte Africaine. ${ }^{131}$

En 2000, la Commission a estimé que la République Fédérale du Nigeria était en violation de divers articles de la Charte Africaine. Il a été constaté que les précautions qui auraient dû être prises pour produire du pétrole, et qui auraient contribué à protéger les droits des victimes de violations signalées, n’ont pas été adoptés. ${ }^{132} \mathrm{La}$ Commission a donc exhorté le gouvernement du Nigeria à assurer la protection de l'environnement, de la santé et des moyens d'existence du peuple Ogoniland. ${ }^{133} \mathrm{Le}$ cas explicité ci-avant est sans aucun doute un témoignage de la précieuse action des ONG devant les quasis juridictions internationales.

127 University of Michigan, Environmental justice case studies by University of Michigan students, disponible sur <http://www.umich.edu/_snre492/cases_03-04/Ogoni/Ogoni_case_study.htm>.

128 Commission Africaine des Droits de l'Homme et des Peuples, 13 - 27 Octobre 2001, Social and Economic Rights Action Center, Center for Economic and Social Rights c. Nigeria, disponible sur <http://www. achpr.org/francais/_doc_target/documentation.html?../decisions/30thsession.pdf . La «Social and Economic Rights Action Center» (Nigeria) et le «Center for Economic and Social Right» (États-Unis) ont exercé leur droit d'actio popularis devant la Commission Africaine.

129 Id., À ce temps là, le Nigeria avait un gouvernement militaire.

$130 \quad I d$

131 Id., Les ONG alléguaient en outre que le gouvernement violait le droit à un environnement sain en:

- participant directement aux activités de contamination de l'air, de l'eau et du sol, nuisant, en ce faisant, à la santé du peuple Ogoniland;

- négligeant de protéger la population Ogoniland contre les torts causés par le Consortium Shell NNPC, et en utilisant plutôt ses forces de sécurité pour faciliter les dommages causés;

- négligeant de fournir ou de permettre la conduite d'études sur les risques éventuels ou réels sur l'environnement et la santé, causés par les activités pétrolières.

132 Id.

133 Id. Suite aux faits survenues à Ogoniland, le gouvernement de Nigeria a crée, pour la première fois dans l'histoire du Nigeria, un Ministère Fédéral de l'Environnement, doté de ressources suffisantes pour aborder les problèmes relatifs à l'environnement qui existent au Nigeria, entre autres mesures. 


\subsection{Les organisations non gouvernementales vis-à-vis des instruments internationaux}

3.3.1. Les ONG vis-à-vis des instruments non contraignants: Le droit à l'environnement sain était, à l'origine, seulement mentionné dans des instruments internationaux de soft law. En réalité, le soft law est un processus souple pour les États leur permettant développer et de tester des normes légales nouvelles avant qu'elles ne deviennent contraignantes dans la communauté internationale. ${ }^{134}$ "The soft law process is more dynamic and democratic than traditional lawmaking, embracing a broader range of actors (including [...] NGOs [...]) and providing a more direct link with the larger society. It has become a critical part of the consensus-building that is ultimately needed to negotiate an environmental treaty». ${ }^{135}$

En fait, les ONG ont joué un rôle essentiel dans le développement de ce type d'instruments, comme nous verrons par la suite. D'ailleurs, il faut relever que la participation des ONG dans ces conférences est le meilleur exemple de la possibilité d'avoir un bon rapport entre les ONG et les organisations internationales.

3.3.1.1. La Déclaration de Stockholm sur l'Environnement Humain: Mis à part quelques traités et décisions spécifiques, le Droit International de l'Environnement ne s'est développé qu’à partir des années septante. Le Droit interne des États a alors commencé à répondre aux impacts négatifs de l'industrialisation ${ }^{136}$. En 1968, la principale préoccupation de la communauté internationale était la pollution transfrontalière de pluie acide. Cette dernière a conduit la Suède à suggérer une conférence internationale pour traiter des problèmes environnementaux. ${ }^{137}$ Ensuite, en 1972, le pays scandinave accepta d'être l'hôte de la Conférence des Nations Unies sur l'Environnement Humain à Stockholm.

En analysant le texte de la déclaration du 16 juin 1972, fruit de la conférence, nous observons que la Déclaration de Stockholm suggère, implicitement, qu'il y a un droit de l'homme à un environnement sain (Principe 1). D'un autre côté, il était encore trop tôt pour que les ONG soient reconnues comme véritables porte-paroles de la société civile, encore moins comme vrais acteurs dans le droit de l'environnement, même si leur participation à cette conférence a été significative. Deux cent cinquante ONG ont été enregistrées. ${ }^{138}$

134 Hunter et as., supra note 77, p. 349.

135 Id.

136 Id., p. 171.

137 Id.

138 Lindblom, supra note 37, pp. 450-451. 
Néanmoins, le bilan après la conférence fut très positif. En effet la Déclaration de Stockholm «a placé les questions écologiques au rang des préoccupations internationales et a marqué le début d'un dialogue entre pays industrialisés et pays en développement concernant le lien qui existe entre la croissance économique, la pollution de l'indivis mondial (l'air, l'eau, les océans) et le bien-être des peuples dans le monde entier». ${ }^{139}$

3.3.1.2. La Déclaration de Rio sur l'Environnement et le Développement: La guerre froide terminée, la Conférence de Rio de 1992 se présentait comme l'opportunité idéale pour que tous les États s'accordent sur une déclaration relative aux deux problématiques les plus sérieuses de l'époque, à savoir: «la détérioration de l'environnement, notamment de sa capacité à entretenir la vie, et l'interdépendance de plus en plus manifeste entre le progrès économique à long terme et la nécessité d'une protection de l'environnement». ${ }^{140}$

A ce sujet, un journaliste de N.Y. Times a écrit ce qu'il suit: «[...] there had never been anything quite like the United Nations Conference on Environment and Development. With 115 heads of state and government attending, it was [...] «the mother of all summits». In all, 178 nations sent some seven thousand delegates to Rio, making it, according to the UN Department of Public Information, the most heavily, reported single event in history». ${ }^{141}$

Révisant le texte de la Déclaration de Rio et essayant de l'interpréter dans une perspective légale, nous pouvons remarquer l'inclusion dans ledit texte de plusieurs principes qui seront considérés plus tard comme faisant partie intégrante du Droit de l'Environnement. Par exemple, le principe de précaution (Principe 15), ${ }^{142}$ l'obligation au niveau national de faire des études d'impact sur l'environnement (Principe 17). ${ }^{143}$

Une différence notable entre la Conférence de Rio et la Conférence de Stockholm fut la grande participation de la société civile à la préparation de Rio. ${ }^{144}$ Plus de 1400 ONG étaient représentées à cette dernière. ${ }^{145}$ Leur participation a contribué à la

\footnotetext{
139 La Déclaration de Rio sur l'Environnement et le Développement (14 juin 1992), disponible sur <http:// www.un.org/french/events/rio92/rio-fp.htm>.

$140 I d$.

141 Id.

142 Ce principe est déjà mentionné à la Convention-Cadre des Nations Unies sur le Changement Climatiques du 09 mai 1992.

143 Les études d'impact sur l'environnement sont une démonstration de l'application du principe de prévention déjà affirmé dans la Convention de Londres de 1972 sur la Prévention de la Pollution des Mers résultant de l'Immersion de Déchets.

144 Hunter et as., supra note 77, p. 196.

145 Id., p. 349.
} 
richesse du texte final. ${ }^{146}$ Cette déclaration a déclenché une montée en puissance des ONG qui a entraîné une "prise de conscience grandissante des responsabilités des citoyens à l'échelle planétaire». ${ }^{147}$

3.3.1.3. La Déclaration de Johannesburg sur le Développement Durable: A la différence des conférences antérieures sur le développement durable ${ }^{148}$ dans lesquelles les délégués étaient exclusivement des représentants de gouvernements, 8,000 représentants de la société civile étaient présents à Johannesburg, ONG comprises. ${ }^{149}$ Par exemple, "Greenpeace was an active participant, making many submissions on a variety of topics throughout the preparatory process and at the Summit itself, as well as providing expert advice towards the process and holding consultations with Nitin Desai $[\ldots]$ and the Summit itself». ${ }^{150}$

Ainsi, le Sommet Mondial pour le Développement Durable a connu une nouvelle manière de dialoguer qui a encouragé une compréhension plus profonde des nécessités de chaque secteur. Le secrétaire général de la conférence, Nitin Desai a, en ce sujet, déclaré "the results of the Summit have been more far comprehensive than any previous outcome. We have put together not only a work plan, but we have identified the actors who are expected to achieve results». ${ }^{151}$ De leur côté, les leaders des ONG ont été aussi satisfaits par la tendance signalée à la Conférence de Johannesburg. ${ }^{152}$

3.3.2. Les ONG vis-à-vis des instruments contraignants: Depuis quelques années, l'engagement des États vis-à-vis de l'environnement est en pleine évolution. Aujourd'hui, ces acteurs ratifient de plus en plus des accords avec d'autres pays dont l'inobservance entraîne l'engagement de leur responsabilité internationale à laquelle sont attachées des sanctions. Nous examinerons de manière sommaire ce fait en prenant quelques conventions comme exemples, constatant au même temps que le rôle des ONG a lui aussi connu un développement.

\footnotetext{
$146 I d$. D'ailleurs, des centaines d'organisations ont participé dans l'alternatif Eco-Forum, réalisé en même temps dans le centre-ville de Rio.

147 Roca, P.J. «Les ONG sont porteuses de sens mais la question de leur identité et de leur représentativité reste posée». In: Le nouvel état du monde: 80 idées-force pour comprendre les nouveaux enjeux internationaux, La Découverte, 2002, p. 113 et s', cité dans Soumy, supra note 10.

148 Voy. Wikypédia L'Encyclopédie Libre, disponible sur <http://fr.wikipedia.org/wiki/D\%C3\%A9 veloppement_durable>. «Le développement durable est, selon la définition proposée en 1987 par la Commission mondiale sur l'environnement et le développement dans le Rapport Brundtland, un développement qui répond aux besoins du présent sans compromettre la capacité des générations futures de répondre aux leurs $[\ldots] »$.

149 Guruswamy, supra note 78, p. 46.

150 GreENPEACE, Earth summit 2002, disponible sur <http://archive.greenpeace.org/earthsummit>.

151 The Johannesburg Test: What will Change?, disponible sur <http://www.johannesburgsummit.org/html/ whats_new/feature_story41.html>, cité dans Guruswamy, supra note 78, pp. 45-46.

152 Guruswamy, supra note 78, p. 46.
} 
3.3.2.1. La Convention sur la Conservation de la Faune et la Flore Marines de l'Antarctique: Les pays signataires de ladite convention ont estimé que la conservation de la faune et la flore marines exigeait une coopération internationale prenant dûment en considération les dispositions du Traité sur l'Antarctique. ${ }^{153}$ Un accord était donc nécessaire afin de permettre la conservation des ressources marines vivantes de l'Antarctique. ${ }^{154}$

Il est évident que le rôle donné aux ONG dans l'accord présent n'est pas significatif. Elles ne sont reconnues ni comme parties signataires, ni comme observateurs. Les ONG sont plutôt considérées comme consultants. ${ }^{155}$ Cependant, en considération de sa date de signature (20 mai 1980), l'inclusion des ONG dans cette convention montre que celles-ci étaient déjà en train de se faire une "place» dans le Droit de l'Environnement.

3.3.2.2. La Convention pour la Protection du Milieu Marin de l'Atlantique du Nord-Est (ci-après "Convention OSPAR»): La Convention OSPAR a pour objet de prévenir et de supprimer la pollution dans la zone maritime européenne. Dans le cadre de cette convention ont été adoptées des mesures plus rigoureuses que celles prévues par les conventions ou accords internationaux de portée mondiale. Cette convention signée en date du 22 septembre 1992 constitue donc une réponse rapide à l'appel émis lors de la Déclaration de Rio afin que les pays coopèrent entre eux dans le domaine de l'environnement.

La Convention OSPAR a été signée par les parties contractantes à la Convention d'Oslo ${ }^{156}$ et à la Convention de Paris ${ }^{157}$ qui s'étaient rendu compte que les conventions citées ne réglementaient pas suffisamment certaines des nombreuses sources de la pollution. ${ }^{158}$ Cette convention est importante parce qu'elle contient un développement profond du principe d'accès à l'information, timidement évoqué lors de

\footnotetext{
153 Le traité sur l'Antarctique fut signé le 1 décembre 1959, il réglemente les relations entre les États signataires en ce qui concerne l'Antarctique. Le traité s'applique aux territoires, incluant les plates-formes glaciaires, situés au sud du $60^{\circ}$ parallèle Sud.

154 La Convention sur la Conservation de la Faune et la Flore Marines de l'Antarctique (20 mai 1980), Art. II, disponible sur <http://www.ccamlr.org/pu/f/f_pubs/bd/ptl.pdf>.

155 Id., Art. XXIII, $\$ 3$. Lowe, supra note 45. Depuis lors, les ONG se sont professionnalisées. "Certaines de ces ONG sont constituées de techniciens chevronnés en matière de droit de l'environnement; d'autres au regard de leurs objectifs spécifiques apparaissent comme des partenaires idoines pour toute réglementation appropriés en certaines matières».

156 La Convention pour la Prévention de la Pollution Marine par les Opérations d'Immersion effectuées par les Navires et Aéronefs a été signée à Oslo le 15 février 1972 et amendée par les protocoles du 2 mars 1983 et du 5 décembre 1989.

157 La Convention pour la Prévention de la Pollution Marine d'Origine Tellurique, a été signée à Paris le 4 juin 1974, et amendée par le protocole du 26 mars 1986.

158 La Convention pour la Protection du Milieu Marin de l'Atlantique du Nord-Est (22 septembre 1992), Préambule, disponible sur <http://www.ospar.org/fr/html/welcome.html>.
} 
la Déclaration de Rio (principe 10). La convention établit également des directives précises sur la pollution transfrontalière. ${ }^{159}$

A la différence de la Convention sur la Conservation de la Faune et la Flore Marines de l'Antarctique dans laquelle les ONG sont reconnues comme consultantes; cette fois-ci la Commission —organe de surveillance de la Convention OSPAR — a la faculté d'admettre en qualité d'observateur toute organisation non gouvernementale «dont les activités ont un rapport avec la Convention». ${ }^{160}$ Ces observateurs peuvent participer aux réunions de la Commission ${ }^{161}$ et soumettre à cet organe toute information ou tout rapport relatif aux objectifs de la Convention. ${ }^{162}$ Actuellement, Greenpeace International, et 32 autres organisations non gouvernementales ont le statut d'observateurs auprès d'OSPAR. ${ }^{163}$

3.3.2.3. Le Protocole de Kyoto à la Convention-Cadre des Nations Unies sur les Changements Climatiques: Depuis la Conférence de Rio, aucun autre sommet environnemental sur l'environnement n'avait attiré autant l'attention que ce que n'ont fait les négociations de Kyoto. ${ }^{164}$

The various positions of the key parties - the European Union, the United States, the G-77- seemed too distant for any meaningful agreement to be reached. [...] Yet the public scrutiny was such that failure to reach and agreement would have been deemed and embarrassing failure to many of the governments. And no one wanted to be blamed for «killing Kyoto». [...] One turning point came when Vice President Al Gore agreed to attend the conference $[\ldots]$ his presence in Kyoto raised the political stakes of failure. ${ }^{165}$

Les négociations sont effectivement arrivées à «bon port». De sorte que les pays signataires de la Convention-Cadre sur les Changements Climatiques soucieux d'atteindre l'objectif ultime de la convention ont signé un accord complémentaire, dit «protocole». Cet objectif consiste à "[...] stabiliser, conformément aux dispositions pertinentes de la Convention, les concentrations de gaz à effet de serre dans l'atmosphère à un niveau qui empêche toute perturbation anthropique dangereuse du système climatique». ${ }^{166}$

159 Id., Art. 21.

160 Id., Art. 11.

161 Id.

162 Id.

163 Commission OSPAR pour la Protection du Milieu Marin de l'Atlantique du Nord-Est, Observateurs et Mémorandums d'Entente, disponible sur <http://www.ospar.org/fr/html/omou/welcome.html>.

164 Hunter et as., supra note 77, p. 629.

$165 \mathrm{Id}$.

166 La Convention-Cadre des Nations Unies sur les Changements Climatiques (9 mai 1992), Art. 2, disponible sur <http://unfccc.int/resource/docs/convkp/convfr.pdf>. 
Les États parties au Protocole sont obligés, conformément aux articles 7 et 8, de donner des informations techniques périodiques à la Conférence des Parties ${ }^{167}$ sur les mesures concrètes prises en vue d'atteindre les objectifs dudit Protocole. Ces informations sont examinées par des équipes composées d'experts. «Les équipes d'examen sont coordonnées par le secrétariat et composées d'experts choisis parmi ceux qui auront été désignés par les parties à la Convention et, le cas échéant, par des organisations intergouvernementales, conformément aux indications données à cette fin par la Conférence des Parties». ${ }^{168}$ En cas d'inobservance des termes de la convention, des sanctions seront imposées. ${ }^{169}$

Dans le cadre du Protocole de Kyoto, la Conférence des Parties sollicite et utilise les services et le concours des organismes non gouvernementaux compétents, ainsi que les informations qu'ils fournissent. ${ }^{170}$ Certaines ONG peuvent donc avoir dans ledit accord un rôle consultatif.

En outre, tout organisme non gouvernemental, national ou international, qui est compétent dans les domaines visés par le présent protocole et qui fait savoir au secrétariat qu'il souhaite être représenté en qualité d'observateur à une session de la Conférence des Parties au présent protocole, peut y être admis en cette qualité. ${ }^{171}$

3.3.2.4. La Convention de la Commission Economique des Nations Unis pour l'Europe sur l'Accès à l'Information, la Participation du Public au Processus Décisionnel et l'Accès à la Justice en matière d'Environnement (ci-après "Convention Aarhus»): Compte tenu du premier principe de la Déclaration de Stockholm sur l'Environnement Humain et du principe 10 de la Déclaration de Rio sur l'Environnement et le

167 Id., Art. 9, 13, 14. La Conférence des Parties, organe suprême de la Convention, fait régulièrement le point de la mise en oeuvre dudit protocole et prend, dans les limites de son mandat, les décisions nécessaires pour en promouvoir la mise en ouvre effective.

168 Id., Art. 8.

169 La mise en place d'un système effectif des sanctions juridiques et/ou matérielles contre les États en cas de non-conformité des conventions internationales doit être une règle dans le domaine de l'environnement.

170 La Convention-Cadre des Nations Unies sur les Changements Climatiques, supra note 166, Art. 13. LindBLOM, supra note 37, p. 461. "The lists of participants in the session of the Conference of Parties present a varied group on non-governmental bodies many attending organizations and bodies were environmental NGOs, trade unions or research institutes».

171 Rien n'interdit dans le texte du Protocole que la même ONG renouvelle sa pétition pour être observateur à chaque session de la Conférence des Parties. C. de Klemm, "Les ONG et les experts scientifiques», in C. IMPERIALI (éd.), supra note 84, p. 85. «Les observateurs n'ont pas le droit de vote, mais ils peuvent participer librement aux discussions. Le règlement intérieur de plusieurs conventions permet, cependant, la tenue de séances à huis clos, hors de la présence des observateurs, mais cela ne se produit que rarement». Greenpeace, Climate summit, Documents, disponible sur <http://weblog.greenpeace.org/climatesummit/documents. html>. En fait, Greenpeace a été impliquée depuis le début dans ce protocole «making submissions, interventions and holding side events and workshops in key issues, with a particular focus on maintaining and enhancing the environmental integrity of the Kyoto Protocol». 
Développement. ${ }^{172}$ Les parties contractantes à la présente convention l'ont signé avec l'intention d'améliorer l'information délivrée par les autorités publiques à propos des problématiques environnementales (telles que la qualité de l'eau); d'étendre des conditions d'accès, d'une part, à la justice en matière de législation environnementale et, d'autre part, à l'information permettant au public de contester des décisions relatives à l'environnement et d'augmenter la participation publique dans la prise de décision en matière de l'environnement (par exemple, sous la forme d'enquêtes publiques). ${ }^{173}$

Cette convention a été adoptée à Aarhus (Danemark) en 1998. Elle a été signée jusqu’à présent par quarante États européens et la Communauté Européenne comme telle. Conformément à l'article 19, $\$ 3$, de ladite Convention, tout État membre de l'ONU peut adhérer à la Convention avec l'accord de la Réunion des Parties. ${ }^{174}$

La Convention Aarhus est importante parce qu'elle confère des droits de caractère procédural aux individus avec pour objectif final de rendre effectif le droit à l'environnement sain. Mais la valeur de cette convention consiste aussi en la participation des ONG depuis son début. ${ }^{175}$

When the actual negotiations began, NGOs were invited by the Chairman [...], to participate on a basis more or less equal to that on which government delegations participated, and availed fully of this opportunity. [...] While from a formal point of view, it might seem anomalous that an observer delegation (le texte fait référence aux ONG) should participate more actively in a negotiating process than voting delegations, the latter (or at least their Chairman) seemed to accept the logic that it would not make sense to negotiate a convention on public participation without a significant measure of participation in the negotiating process. ${ }^{176}$

\footnotetext{
172 Convention sur l'Accès à l'Information, la Participation du Public au Processus Décisionnel et l'Accès à la Justice en matière d'Environnement (25 juin 1998), Préambule, disponible sur <http://www.centre.environnement.gouv.fr/telechargement/la_convention_aarhus.htm>.

173 Europa Le Portail de l'Union Européenne, Environnement, Dispositions générales, Convention Aarhus, disponible sur <http://europa.eu/scadplus/leg/fr/lvb/l28056.htm>.

174 Convention on Access to Information, Public Participation in Decision-Making and Access to Justice in Environmental Matters, disponible sur <http://www.unece.org/env/pp/ctreaty.htm>. Voy. Europa Gateway to the European Union, European Commision, Environment, Working Together, Aarhus, disponible sur $<$ http://ec.europa.eu/environment/aarhus/index.htm\#legislation>. La Convention de Aarhus a été adoptée par la Communauté Européenne le 17 février 2005 via la Décision 2005/370/EC. La Communauté est partie à la convention depuis mai 2005. La Convention devait être appliquée dans les États membres au plus tard le 25 juin 2005, concernant l'accès au public à l'information environnementale (Directive 2003/4/EC) et la participation du public dans la prise de décisions à ce sujet (Directive 2003/35/EC). Quant à l'accès du public à la justice permettant lui de contester des décisions relatives à l'environnement, l'implémentation a été ordonnée par le Parlement Européen via le Règlement No 1367/2006. Ce Règlement "covers not only the institutions, but also bodies, offices or agencies established by, or on the basis of the EC Treaty. All those will have until 28 June 2007 to adapt their internal procedures and practice to the provisions of the Regulation».

175 Wates, J. «NGOs and the Aarhus convention». In TREves et as. (éd.), supra note 87, p. 177.

176 Id., p. 178.
} 
En effet, les ONG ont profité au maximum de cette opportunité dans l'élaboration du texte de la Convention Aarhus en travaillant en coalition, toutes réunies dans le Forum Européen d'organisations environnementales de citoyens et présentant des positions coordonnées dans chaque point de discussion. ${ }^{177}$ Ceci étant, la participation des ONG a continué bien au-delà, c'est-à-dire jusqu'à la phase d'implémentation de la Convention. Elles ont participé aux réunions de pays signataires et se sont impliquées dans chaque groupe de travail constitué pour préparer la première Réunion des Parties. ${ }^{178}$

Larticle $10, \$ 5$ octroie à toute organisation non gouvernementale ayant un rapport au cadre de la présente convention le droit de participer en qualité d'observateur aux Réunions des Parties. ${ }^{179}$

Several other decisions of the Meeting of the Parties specify a role for the public or NGOs in the respective processes under the Convention. Perhaps the most significant references providing for the involvement of NGOs are those in decision I/7. ${ }^{180}$

La décision I/7, prise à la Premiere Reunion de Parties, établit un Comité en charge de l'application des termes de cette convention: «consisting of eight members serving in a personal capacity (rather than as representative of parties). These individual are to be national of parties or signatories to the Convention $[\ldots] » .{ }^{181}$ Cependant, cette décision stipule que les ONG qui remplissent les conditions de l'article 10 de la convention peuvent également élire des candidats pour le Comité. Bien que la Réunion des Parties nomme des candidats pour le Comité, sans obligation d'élire des candidats proposés par les ONG, le droit des organisations non gouvernementales est quand même significatif. ${ }^{182}$ De plus, le Comité est ouvert aux personnes naturelles

\footnotetext{
177 Id. Malheureusement, la pratique révèle que «le fait pour une ONG de participer étroitement à la rédaction d'un texte et à la création d'un mécanisme de contrôle ou de sanction n'emporte pas nécessairement l'action des ONG devant ces mécanismes de contrôle». Breton-Legoff, supra note 4.

178 WATES, supra note 175 , p. 179.

179 Lowe, supra note 45. Comme nous pouvons voir, la règle actuelle est que: «les organisations internationales compétentes en matière de protection de l'environnement admettent les représentants d'ONG à titre d'observateurs, ce rôle est plus important qu'il ne le paraît. [...] De plus, les ONG peuvent ne serait- ce qu'à titre d'observateurs nouer des contacts avec le secrétariat et les parties à la convention. Une telle situation est avantageuse dans la mesure où les études, rapports ou propositions des ONG pourraient permettre d'influencer les décisions des parties contractantes».

180 WaTES, supra note 175 , p. 180.

181 Id.

182 Id., p. 181. A titre d'exemple, comme nous avons indiqué au point 2.3.2.3 le Protocole à la Convention de Kyoto affirme que c'est une équipe d'experts, nommé par le Secrétariat, qui évalue les rapports périodiques des pays membres. Pourquoi ne pas inclure dans cette équipe d'autres experts nommés par l'ensemble des ONG spécialistes en la matière ou, en tout cas, proposées par celles-ci? Cela apporterait plus d'indépendance, de neutralité et d'expertise aux équipes d'examen. Une option semblable a été choisie dans l'Accord relatif au Programme International pour la Conservation des Dauphins de 1998; il prévoit «[...] for an International Review Panel (IRP), made up not only of representatives of the parties, but also three representatives from
} 
ou morales, y compris les ONG. Elles peuvent donc présenter des communications sur l'inapplication des termes du traité devant le Comité. ${ }^{183}$

D'ailleurs, à la Deuxième Réunion des Parties on a décidé d'adopter les Lignes Directrices d'Almaty. Leur objectif principal est de fournir des orientations générales aux parties sur les moyens de promouvoir l'application des principes de la Convention d'Aarhus dans les instances internationales pour les questions touchant à l'environnement. ${ }^{184}$ A cette fin, il s'est accordé, entre autres, de renforcer des capacités des ONG oeuvrant en faveur de la protection de l'environnement, tout particulièrement dans les pays en développement et les pays en transition; ${ }^{185}$ 's'exhorte aux parties d'autoriser les ONG d'attendre aux réunions des instances internationales relatives à des questions liées à l'environnement, à tous les stades pertinents du processus décisionnel, y compris au niveau des organes subsidiaires. ${ }^{186}$ De plus, il est invoqué aux parties d'entreprendre des efforts pour recourir à des approches innovantes, rentables et pratiques, de façon à assurer la plus large participation possible des ONG aux instances internationales. ${ }^{187}$

\section{L'ancien Secrétaire Général de l'ONU Kofi Annan a souligné le caractère novateur et original de la convention en ces termes:}

Although regional in scope, the significance of the Aarhus Convention is global. It is by far the most impressive elaboration of principle 10 of the Rio Declaration, which stresses the need for citizens' participation in environmental issues and for access to information on the environment held by public authorities. As such, it is the most ambitious venture in the area of environmental democracy so far undertaken under the auspices of the United Nations. ${ }^{188}$

\footnotetext{
experienced environmental NGOs and three representatives from the affected tuna industry. IRP responsibilities include, inter alia, analysis of reports submitted to the IRP regarding fishing by vessels covered by the Agreement, identification of possible infractions of the Agreement, and coordination with the party whose flag the vessel flies of possible infractions and any enforcement actions taken with respect to that vessel». Hollis, supra note 19.

183 Lindblom, supra note 37, p. 286.

184 La décision qui établit les Lignes Directrices d'Almaty a été pris le 20 juin 2005 à Almaty (Kazakhstan). Lignes Directrices d'Almaty, point 1, disponible sur <http://www.unece.org/env/documents/2005/pp/ece/ ece.mp.pp.2005.2.add.5.f.pdf>.

185 Lignes Directrices d'Almaty, point 17. Les Lignes directrices attribuent ces droits aux ONG parce que les conventions qui oeuvrent en faveur de la protection de l'environnement et qui remplissent les conditions pouvant être requises en droit interne sont réputées avoir un intérêt à agir dans le cadre de la convention.

186 Id., point 29.

187 Id., point 39.

188 United Nations Economic Commission for Europe, Aarhus Convention, disponible sur <http://www. unece.org/env/pp/s.
} 


\section{Conclusions}

Le droit de l'environnement, inexistant avant la première moitié du siècle passé, a connu un développement extraordinaire qui, sans doute, s’accéléra considérablement avec la pleine reconnaissance du droit à un environnement sain comme droit de l'homme. Ce qui est apparu comme une hypothèse n'est que confirmée dans les faits. Si les pays s'engagent aujourd'hui de plus en plus dans des instruments contraignants au niveau régional et universel, et destinent des fonds publics pour différents projets de défense de l'écologie, ce n'est pas uniquement de leur propre gré. C'est principalement grâce au travail quotidien des ONG que les autorités étatiques s'attèlent plus ardemment à promouvoir la protection, la préservation et l'amélioration de l'environnement.

En reconnaissance de la contribution des ONG au développement du droit de l'environnement, les États leur octroient des prérogatives en Droit International, ce qui montre en même temps l'évolution de celles-ci. Après avoir été ignorées dans les comités de préparation des conférences internationales, les ONG sont devenues le «moteur» de ces événements; elles sont passées d'entités sans aucun pouvoir devant des conventions internationales, au statut d'observateurs. Après avoir été de simples spectatrices vis-à-vis des instruments internationaux, les ONG interviennent dans le libellée des traités; d'amicus curiae, elles ont acquis la position de requérants devant des cours supranationales; et récemment les ONG commencent à remplir une fonction plus importante encore, être garantes du stricte respect et exécution des termes des instruments internationaux. Malheureusement, même si nous pouvons illustrer ce que nous venons d'affirmer, il s'agit de cas ponctuels. Transformons ces exceptions en règle!

Il y a donc des tâches à réaliser. Par exemple, des mesures législatives deviennent impératives comme celle de la Belgique ou des accords internationaux comme la Convention Européenne sur la Reconnaissance de la Personnalité Juridique des Organisations Internationales Non Gouvernementales, les deux permettant aux ONG de jouir des mêmes droits dans un pays tiers que ceux déjà reconnus dans le pays du premier enregistrement.

L'inclusion des organisations non gouvernementales dans le processus de négociations et le libellé des instruments internationaux non contraignants a été essentielle pour la richesse des termes desdites déclarations. Une participation majeure des ONG, cette fois-ci dans l'élaboration des instruments de hard law s'impose car, vu les conditions actuelles de notre planète, il faut s'efforcer de trouver directement un consensus en la matière sans passer par des tests permis avec le soft law. 
L'actio popularis a prouvé son effectivité dans le cas de la Commission Africaine de Droits de l'Homme et des Peuples. Des exemples comme ceux-ci doivent être suivis par des «vraies» juridictions internationales. Ainsi, la Cour Africaine des Droits de l'Homme et des Peuples devrait enlever la restriction selon laquelle les ONG peuvent saisir ladite cour uniquement avec l'autorisation de l'État concerné. De même, la Cour Européenne des Droits de l'Homme devrait modifier sa procédure et permettre la défense des intérêts collectifs via ce mécanisme procédural, en sachant que les victimes de la violation du droit à un environnement sain sont -presque toujours- les populations rurales les plus pauvres qui n'ont pas accès à l'information sur les juridictions internationales, encore moins les moyens économiques pour les supporter.

La mise en pratique des propositions comme la création d'une organisation internationale de l'environnement ou d'une cour internationale dans ce domaine-là, est un sujet dont la pertinence mérite d'être étudiée sérieusement.

L'expérience et les connaissances techniques des ONG méritent de profiter au maximum aux organisations internationales et aux États. Par conséquent, il est nécessaire de chercher des mesures créatives pour une participation plus active des ONG dans l'application des accords et/ou programmes internationaux comme celle établie par la Convention de Aarhus. La survie des générations futures est en péril. Faciliter le travail des ONG de la part des États se présente donc comme une obligation qui deviendra bénéfique pour tous. 